\title{
Diagnostics of isopycnal mixing in a circumpolar channel
}

\author{
Ryan Abernathey ${ }^{\mathrm{a}, *}$, David Ferreira ${ }^{\mathrm{b}}$, Andreas Klocker ${ }^{\mathrm{c}, \mathrm{d}}$ \\ ${ }^{a}$ Scripps Institution of Oceanography, 8820 Shellback Way, La Jolla, CA, United States \\ ${ }^{\mathrm{b}}$ Massachusetts Institute of Technology, 77 Massachusetts Ave, Cambridge, MA 02139, United States \\ ${ }^{\mathrm{c}}$ The Australian National University, Barry Drive, Acton ACT 0200 ACT, Australia \\ ${ }^{\mathrm{d}}$ The Australian Research Council, Center of Excellence for Climate System Science, Australia
}

\section{A R T I C L E I N F O}

\section{Article history:}

Received 25 February 2013

Received in revised form 20 July 2013

Accepted 26 July 2013

Available online 8 August 2013

\section{Keywords:}

Mesoscale eddies

Eddy diffusivity

Isopycnal mixing

Antarctic Circumpolar Current

\begin{abstract}
A B S T R A C T
Mesoscale eddies mix tracers along isopycnals and horizontally at the sea surface. This paper compares different methods of diagnosing eddy mixing rates in an idealized, eddy-resolving model of a channel flow meant to resemble the Antarctic Circumpolar Current. The first set of methods, the "perfect" diagnostics, are techniques suitable only to numerical models, in which detailed synoptic data is available. The perfect diagnostic include flux-gradient diffusivities of buoyancy, QGPV, and Ertel PV; Nakamura effective diffusivity; and the four-element diffusivity tensor calculated from an ensemble of passive tracers. These diagnostics reveal a consistent picture of isopycnal mixing by eddies, with a pronounced maximum near $1000 \mathrm{~m}$ depth. The isopycnal diffusivity differs from the buoyancy diffusivity, a.k.a. the Gent-McWilliams transfer coefficient, which is weaker and peaks near the surface and bottom. The second set of methods are observationally "practical" diagnostics. They involve monitoring the spreading of tracers or Lagrangian particles in ways that are plausible in the field. We show how, with sufficient ensemble size, the practical diagnostics agree with the perfect diagnostics in an average sense. Some implications for eddy parameterization are discussed.
\end{abstract}

() 2013 Elsevier Ltd. All rights reserved.

\section{Introduction}

Mesoscale eddies play an important role in the transport of heat, salt, potential vorticity, and carbon in the ocean, particularly in the Southern Ocean (Marshall and Speer, 2012; Lauderdale et al., 2013). Eddy transport can be described as the sum of an advective flux and a diffusive flux along isopyncals (Redi, 1982; Griffies, 1998). Motivated by the importance of eddy fluxes, much recent research has focused on characterizing the mixing properties of mesoscale eddies in the Southern Ocean (Marshall et al., 2006, 2008, 2009,a,b, 2010, 2011, 2010,, 2012a,b,). A field campaign to measure mixing rates, the Diapycnal and Isopycnal Mixing Experiment in the Southern Ocean (a.k.a. DIMES; Gille et al., 2012), is also underway.

Since eddy fluxes are so difficult to measure directly on a large scale, the hope underlying these efforts is that better knowledge of the eddy mixing rates will allow us to infer the eddy fluxes through diffusive closures. However, a wide range of mixing diagnostics have been employed, and the link between such diagnostics of mixing and the actual eddy-induced transport is somewhat obscure. A further complication is that the (Gent and McWilliams,

\footnotetext{
* Corresponding author. Address: Lamont-Doherty Earth Observatory, 205 C Oceanography, 61 Route 9W-PO Box 1000, Palisades, NY 10964-8000, United States. Tel.: +1 6178004236 .

E-mail address: rpa@ldeo.columbia.edu (R. Abernathey).
}

1990) eddy transfer coefficient, which is necessary for coarse-resolution models to parameterize eddy-induced advection, is not related to the eddy diffusivity in a simple way (Smith and Marshall, 2009).

The goal of this paper is to directly compare various methods of diagnosing isopycnal mixing. Some of these diagnostics are possible only in the context of a numerical model, in which all the dynamical fields are known exactly. We call these "perfect" diagnostics. We also consider less precise diagnostics which can potentially be applied to the real ocean, for example, in DIMES. We call these "practical" diagnostics.

This study builds on many previous works, beginning with Plumb and Mahlman (1987), who first proposed the method for inferring $\mathbf{K}$, the eddy diffusivity tensor, in an atmospheric model. A comparison between the diffusivities of passive tracers, potential vorticity, and buoyancy was performed by Treguier (1999) in a primitive-equation model and later in a quasi-geostrophic model by [henceforth SM09] Smith and Marshall (2009). Our study builds on their approach by using primitive equations, including a more realistic residual meridional overturning circulation, and by calculating diffusivities as functions of $y$ and $z$, rather than $z$ alone. Marshall et al. (2006), Abernathey et al. (2010), Ferrari and Nikurashin (2010) and Lu and Speer (2010) all calculated "effective diffusivity" based on the method of Nakamura (1996), but did not compare their calculations to other mixing diagnostics. Klocker et al. 
(2012a) demonstrated the equivalence between tracer and particle-based diffusivities, but did so only in a 2D flow; here we work in three dimensions. In summary, the program of this paper is to synthesize and summarize these disparate methods in a flow with a plausible meridional overturning circulation, and then to compare them with the less precise methods available in the field.

Our central conclusion is that disparate methods do in fact give reasonably similar results; we find roughly the same diffusivities for passive tracers, Lagrangian floats, quasigeostrophic potential vorticity, and planetary Ertel potential vorticity. These all have similar magnitudes and vertical structures, with a pronounced mid-depth maximum. But, as previously reported by Treguier (1999) and SM09, none of them is very similar to the Gent-McWilliams coefficient, which has a lower magnitude and different vertical structure.

\section{Numerical model}

The model flow is meant to resemble the Antarctic Circumpolar Current. The domain, numerical configuration, and forcing are identical to the model described in Abernathey et al. (2011) and Hill et al. (2012), which the reader should consult for a detailed description.

The Boussinesq primitive equations are solved using the MITgcm (Marshall et al., 1997; Marshall et al., 1997). The domain is a zonally reentrant channel on a $\beta$-plane, of dimensions $L_{x} \times L_{y} \mathrm{x}$ $H$, where $L_{x}=1000 \mathrm{~km}, L_{y}=2000 \mathrm{~km}$, and $H=2985 \mathrm{~m}$. It is forced at the surface with a zonal wind stress and a fixed heat flux. The forcing and domain, along with a snapshot of the temperature field, are illustrated in Fig. 1. The wind stress forcing is a sinusoid which peaks in the center of the domain at $0.2 \mathrm{~N} \mathrm{~m}^{-2}$. The heat flux consists of sinusoidally alternating regions of cooling, heating, and cooling, with an amplitude of $10 \mathrm{~W} \mathrm{~m}^{-2}$. There is a sponge layer at the northern boundary, in which the temperature is relaxed to an exponential stratification profile with an e-folding scale of $1000 \mathrm{~m}$. A second-order-moment advection scheme is used to minimize spurious numerical diffusion (Prather, 1986), resulting in an effective diapycnal diffusivity of approx. $10^{-5} \mathrm{~m}^{2} \mathrm{~s}^{-1}$ (Hill et al., 2012). The model contains no salt and uses a linear equation of state; the buoyancy is simply $b=g \alpha_{T} \theta$, where $g$ is gravity, $\alpha_{T}$ is the constant thermal expansion coefficient, and $\theta$ is the potential temperature.

The fine resolution ( $5 \mathrm{~km}$ in the horizontal, 40 vertical levels), together with the forcing, which maintains a baroclinically unstable background state, allows an energetic mesoscale eddy field to develop. Without the sponge layer, the eddy-induced overturning circulation would nearly cancel the wind-driven Eulerian-mean overturning circulation, resulting in a very small residual overturning circulation, a situation described by Kuo et al. (2005). However, the presence of the sponge layer, in conjunction with the applied pattern of heating and cooling, produces a residual overturning that qualitatively resembles the real Southern Ocean, as described by Marshall and Radko (2003) or Lumpkin and Speer (2007) (see for further detail Abernathey et al., 2011).

This residual overturning circulation is obtained by averaging the meridional transport $v$ in layers of constant buoyancy $b$; the streamfunction obtained this way is defined as

$\Psi_{i s o}(y, b)=\frac{1}{\Delta t} \int_{t_{0}}^{t_{0}+\Delta t} \iint_{-D}^{0} v \mathcal{H}(b) d z d x d t$

where $\mathcal{H}$ is the heaviside function and $D$ is the depth. In Fig. 1 we plot $\Psi_{\text {iso }}$ in its native buoyancy coordinates and also mapped back into depth coordinates. The figure reveals two distinct cells: a coun-
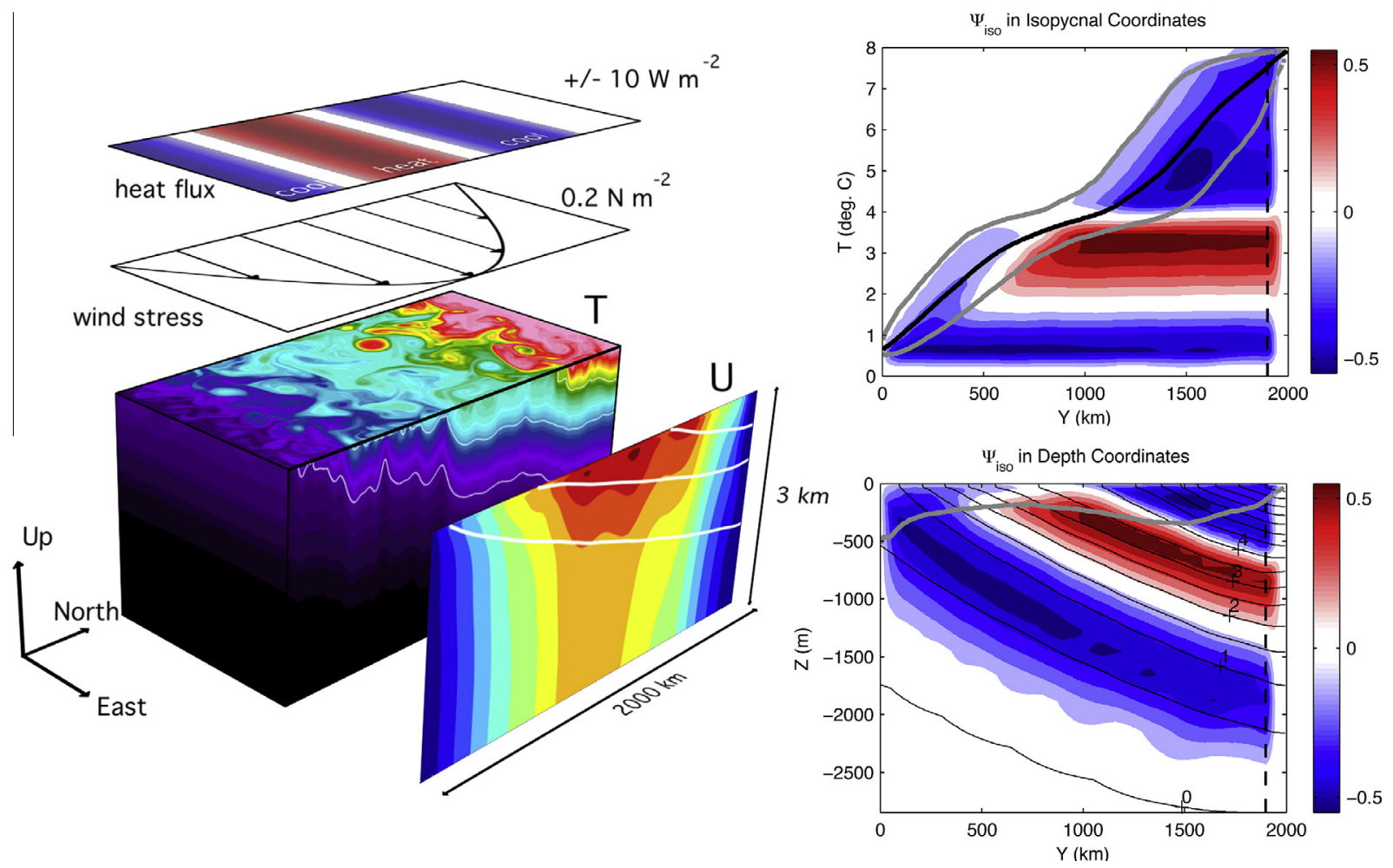

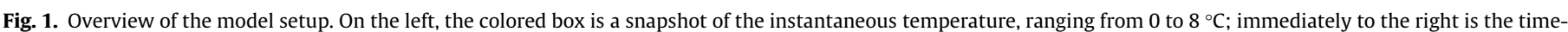

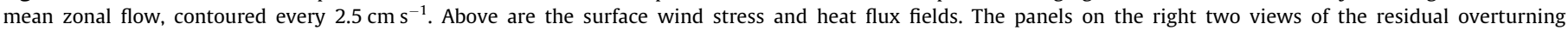

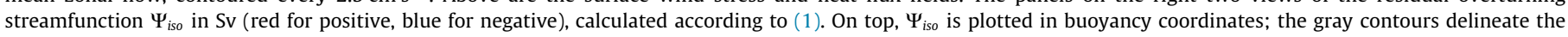

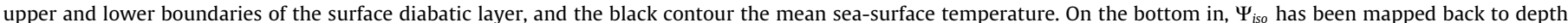

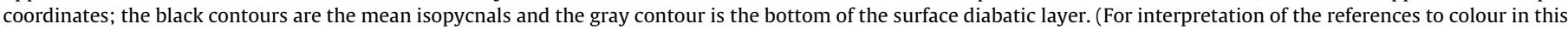
figure caption, the reader is referred to the web version of this article.) 
terclockwise lower cell, analogous to the Antarctic-Bottom-Water branch of the global MOC (Ito and Marshall, 2008); and a clockwise mid-depth cell, analogous to the upper branch of the global MOC (Marshall and Speer, 2012). There is also a shallow subduction region in the north of the domain that can be viewed as a mode-water formation region.

The fact that our model has non-zero interior residual circulation also implies that there are non-zero gradients and eddy fluxes of potential vorticity (PV) in the interior. These PV fluxes are directly related to the residual transport (Andrews et al., 1987; Plumb and Ferrari, 2005). The presence of non-zero interior PV is a key property that allows us to demonstrate the similarity in the mixing of dynamically passive tracers and floats to the dynamically active mixing of PV. In the following sections, the velocity field from the equilibrated model will be used to advect passive tracers and particles.

It should be noted that, because our model has no topography, the wind stress is balanced by bottom frictional drag rather than topographic form drag. This means that the model has a very large barotropic zonal mean flow, leading to an unrealistically large zonal transport (approx. $800 \mathrm{~Sv}$ ). The thermal-wind induced transport, however, is much more realistic (approx. $100 \mathrm{~Sv}$ ). Given the known importance of the mean flow in suppressing meridional mixing in the ACC (Abernathey et al., 2010; Ferrari and Nikurashin, 2010), it is reasonable to ask whether this flow will affect the measured mixing rates. In fact, we do not expect this unrealistic zonal transport to affect our results substantially. This is because the suppression factor due to the mean flow is proportional to $(U-c)^{2}$, where $U$ is the mean zonal velocity and $c$ is the eddy phase speed (Ferrari and Nikurashin, 2010). The addition of a barotropic mean flow translates the eddies along with it, augmenting $U$ and $c$ similarly (Klocker and Marshall, 2013, manuscript submitted to $J$. Phys. Oceanogr.). It is the relative propagation that depends on the PV gradient. In the simplest case, consider a barotropic Rossby wave in the presence of a mean flow: the dispersion relation is $U-c=\beta / k^{2}$ where $\beta$ is the planetary vorticity gradient and $k$ is the wavenumber. In our case, the dispersion relation is more complex, but the same principle applies.

The great advantage of using a domain without topography is the zonal symmetry, which permits us to focus only on meridional mixing rates, rather than the much more difficult problem of twodimensional mixing. Indeed many of our diagnostics (e.g. $\left.K_{\text {eff }}\right)$ cannot be applied locally in two dimensions. The zonal average also serves to eliminate the contribution of rotational fluxes, which can contaminate the down-gradient nature of the eddy flux (Marshall and Shutts, 1981).

\section{Perfect mixing diagnostics}

The "perfect" mixing diagnostics are quantities which can be calculated only with very detailed synoptic knowledge of the flow. Such diagnostics provide the most complete characterization of mixing and transport possible. They are straightforward to extract from numerical models but nearly impossible for the real ocean. By contrast, in the atmosphere, some perfect diagnostics can be calculated directly from observations (e.g.Nakamura and Ma, 1997) or from reanalysis products (e.g.Haynes and Shuckburgh, 2000a; Haynes and Shuckburgh, 2000b).

Observational problems aside, the interpretation of perfect mixing diagnostics still poses a challenge. Different diagnostics have been used throughout the literature to characterize eddy mixing, and the relationship between these diagnostics is not always obvious. Our purpose here is to consolidate many different diagnostics in one place and show their relationship. A similar study was made for the atmosphere by Plumb and Mahlman (1987) hereafter
PM87, who also review some theoretical aspects. Here we basically repeat their methodology for this ACC-like flow.

Below each diagnostic is described and discussed individually. A summary comparison of all the perfect isopycnal diffusivities can be found in the discussion at the end of this section (Section 3.3) and in Fig. 8.

\subsection{Passive tracers}

Our starting point is to examine the mixing of passive tracers. Passive tracers obey an advection-diffusion equation of the form

$\frac{\partial c}{\partial t}+\boldsymbol{v} \cdot \nabla c=\kappa \nabla^{2} c+C$,

where $c$ is the tracer concentration, $\boldsymbol{v}$ is the velocity field, $\kappa$ is a small-scale diffusivity, and $C$ is a source or sink. We will focus on cases where $C=0$ and the diffusive term is negligible for the large-scale budget of $c$. (Some small-scale diffusion is necessary for mixing to occur, and likewise it is impossible to eliminate diffusion completely from numerical models. But for flows of large Péclet number, diffusion is an important term only in the tracer variance budget, not the mean tracer budget itself.)

\subsubsection{Diffusivity tensor}

PM87 performed a detailed study of the transport characteristics of a model atmosphere using passive tracers. Here we briefly review their definition of $\mathbf{K}$, the diffusivity tensor, which we view as the most complete diagnostic of eddy transport. The reader is referred to Plumb and Mahlman, 1987 or Bachman and Fox-Kemper (2013) for a more in-depth discussion.

Taking a zonal average of (2) (indicated by an overbar) and neglecting the RHS terms, we obtain

$\frac{\partial \bar{c}}{\partial t}+\overline{\boldsymbol{v}} \cdot \nabla \bar{c}=-\nabla \cdot \boldsymbol{F}_{c}$,

where $\boldsymbol{F}_{c}=\left(\overline{v^{\prime} C^{\prime}}, \overline{W^{\prime} C^{\prime}}\right)$ is the eddy flux of tracer in the meridional plane. The diffusivity tensor $\mathbf{K}$ relates this flux to the background gradient in each direction; it is defined by

$\boldsymbol{F}_{c}=-\mathbf{K} \cdot \nabla \overline{\boldsymbol{c}}$.

This equation is underdetermined for a single tracer, but PM87 used multiple tracers with different background gradients to calculate it. This method has also recently been applied by Bachman and FoxKemper (2013) in an oceanic context.

We found $\mathrm{K}$ by solving (4) for six independent tracers. In this case, (4) is overdetermined, and the "solution" is a least-squares best fit (Bratseth, 1998; Bachman and Fox-Kemper, 2013). The initial tracer concentrations used were as follows: $c_{1}=y, c_{2}=z, c_{3}=\cos \left(\pi y / L_{y}\right) \cos (\pi z / H), c_{5}=\sin \left(\pi y / L_{y}\right) \sin (\pi z / H)$, $c_{5}=\sin \left(\pi y / L_{y}\right) \sin (2 \pi z / H), c_{6}=\cos \left(2 \pi y / L_{y}\right) \cos (\pi z / H)$. (We experimented with different initial concentrations, but found the results to be insensitive to this detail, provided many tracers with different gradients were used.) The tracers were allowed to evolve from these initial conditions for one year. (An experiment with two years of evolution produced very similar results.) $\boldsymbol{F}_{c}$ and $\nabla \bar{c}$ were calculated for each tracer by performing a zonal and time average over the one-year period and then over an ensemble of 20 different years. In matrix form, the equation solved to find $\mathbf{k}(y, z)$ was

$$
\begin{aligned}
& {\left[\begin{array}{llll}
\overline{v^{\prime} c_{1}^{\prime}} & \overline{v^{\prime} c_{2}^{\prime}} & \ldots & \overline{v^{\prime} c_{6}^{\prime}} \\
\overline{w^{\prime} c_{1}^{\prime}} & \overline{W^{\prime} c_{2}^{\prime}} & \ldots & \overline{w^{\prime} c_{6}^{\prime}}
\end{array}\right]} \\
& =-\left[\begin{array}{ll}
K_{y y} & K_{y z} \\
K_{z y} & K_{z z}
\end{array}\right]\left[\begin{array}{llll}
\partial \overline{c_{1}} / \partial y & \partial \overline{c_{2}} / \partial y & \ldots & \partial \overline{c_{6}} / \partial y \\
\partial \overline{c_{1}} / \partial z & \partial \overline{c_{2}} / \partial z & \ldots & \partial \overline{c_{6}} / \partial z
\end{array}\right],
\end{aligned}
$$

where each element of $\mathrm{K}$ at each point in $(y, z)$ space is a leastsquares estimate that minimizes the error across all tracers. In 
general the fit is very good, with $R^{2}>0.99$ in much of the domain and $R^{2}>0.9$ nearly everywhere. A more detailed discussion of the errors involved in the diffusivity inversion can be found in Appendix A.

It is most informative to decompose $\mathbf{K}$ into two parts,

$\mathbf{K}=\mathbf{L}+\mathbf{D}$,

where $\mathbf{L}$ is an antisymmetric tensor and $\mathbf{D}$ is symmetric. Because the flux due to $\mathbf{L}$ is normal to $\nabla \bar{c}$, its effects are advective, rather than diffusive (Plumb, 1979; Plumb and Mahlman, 1987; Griffies, 1998). Using this fact, we can rewrite (3) as

$\frac{\partial \bar{c}}{\partial t}+\left(\overline{\boldsymbol{v}}+\boldsymbol{v}^{\dagger}\right) \cdot \nabla \overline{\boldsymbol{c}}=\nabla \cdot(\mathbf{D} \cdot \nabla \overline{\boldsymbol{c}})$

where $\boldsymbol{v}^{\dagger}=\left(v^{\dagger}, w^{\dagger}\right)$ is an eddy-induced effective transport velocity, defined by a streamfunction $\chi$, such that

$v^{\dagger}=-\partial \chi / \partial z, \quad w^{\dagger}=\partial \chi / \partial y$

and

$\mathbf{L}=\left[\begin{array}{ll}0 & -\chi \\ \chi & 0\end{array}\right]$

Under adiabatic conditions, $\chi$ is approximately equal to the transformed-Eulerian-mean eddy-induced streamfunction, or the "bolus transport" streamfunction in thickness-weighted isopycnal coordinates. Again, for more detailed discussion, the reader is referred to PM87.

Because $\mathbf{L}$ is advective in nature (and does not appear in the tracer variance budget), all of the actual mixing due to eddies is contained in D (Nakamura, 2001). Since $\mathbf{D}$ is symmetric, it can be diagonalized by coordinate rotation. Let $\mathbf{U}_{\alpha}$ be the rotation matrix for angle $\alpha$. In the rotated coordinate system, the flux due to $D$ is

$-\mathbf{U}_{\alpha} \mathbf{D} \nabla \overline{\boldsymbol{c}}=-\mathbf{U}_{\alpha} \mathbf{D} \mathbf{U}_{\alpha}^{T} \mathbf{U}_{\alpha} \nabla \overline{\boldsymbol{c}}=-\mathbf{D}^{\prime} \mathbf{U}_{\alpha} \nabla \overline{\boldsymbol{c}}$

where $\mathbf{D}^{\prime}=\mathbf{U}_{\alpha} \mathbf{D} \mathbf{U}_{\alpha}^{T}$. Solving for the $\alpha$ that makes $\mathbf{D}^{\prime}$ diagonal, we find

$\tan 2 \alpha=\frac{2 D_{y z}}{D_{y y}-D_{z z}}$.
The rotated matrix,

$\mathbf{D}^{\prime}=\left[\begin{array}{ll}D_{y y}^{\prime} & 0 \\ 0 & D_{z z}^{\prime}\end{array}\right]$

describes the eddy diffusion along ( $D_{y y}^{\prime}$, the major-axis diffusivity) and across ( $D_{z z}^{\prime}$, the minor-axis diffusivity) the plane defined by $\alpha$, which we call the mixing angle. For small $\alpha$, it is convenient to approximate $\alpha \simeq D_{y z} / D_{y y}, D_{y y}^{\prime} \simeq D_{y y}$, and $D_{z z}^{\prime} \simeq D_{z z}-D_{y z}^{2} / D_{y y}$.

The physical interpretation of $\mathbf{K}$ is therefore best summarized by four quantities: $\chi, \alpha, D_{y y}^{\prime}$, and $D_{z z}^{\prime}$. The most relevant for this study, which is concerned with isopycnal mixing, are $D_{y y}^{\prime}$ and $\alpha$, the major axis diffusivity and the mixing angle, which are plotted in Fig. 2. From this figure, we see that the mixing angle is along isopycnals throughout most of the domain, except close the surface, where the mixing acquires a more horizontal character. This pattern is consistent with the paradigm that ocean eddies mix adiabatically in the interior and diabatically in the "surface diabatic layer," i.e. the layer over which isopycnals outcrop (Treguier et al., 1997; Cerovecki and Marshall, 2008). Consequently, $D_{y y}^{\prime}$ can be described as an isopycnal eddy diffusivity in most of the interior. Because of the small aspect ratio, and consequently small $\alpha, D_{y y}^{\prime} \simeq D_{y y}$ is a very good approximation.

An obvious feature in the spatial structure of $D_{y y}^{\prime}$ is a pronounced peak at mid-depth (approx. $1200 \mathrm{~m}$ ). Enhanced isopycnal mixing at a mid-depth "critical layer" is a general feature of baroclinically unstable jets (Green, 1970; Killworth, 1997). Many studies have confirmed the presence of an enhanced mid-depth mixing layer in the ACC (Smith and Marshall, 2009; Abernathey et al., 2010; Naveira-Garabato et al., 2011; Klocker et al., 2012a). Our highly idealized model evidently shares this behavior. It is also important to note, though, that $D_{y y}^{\prime}$ varies even more strongly with $y$, with the strongest mixing being in the center of the channel.

\subsubsection{Eddy-induced advection}

The streamfunction $\chi$, derived from the anti-symmetric part of $\mathbf{K}$, describes an eddy-induced advective transport in the meridional plane. For statistically steady, adiabatic conditions, this circulation is expected to approximately equal both the

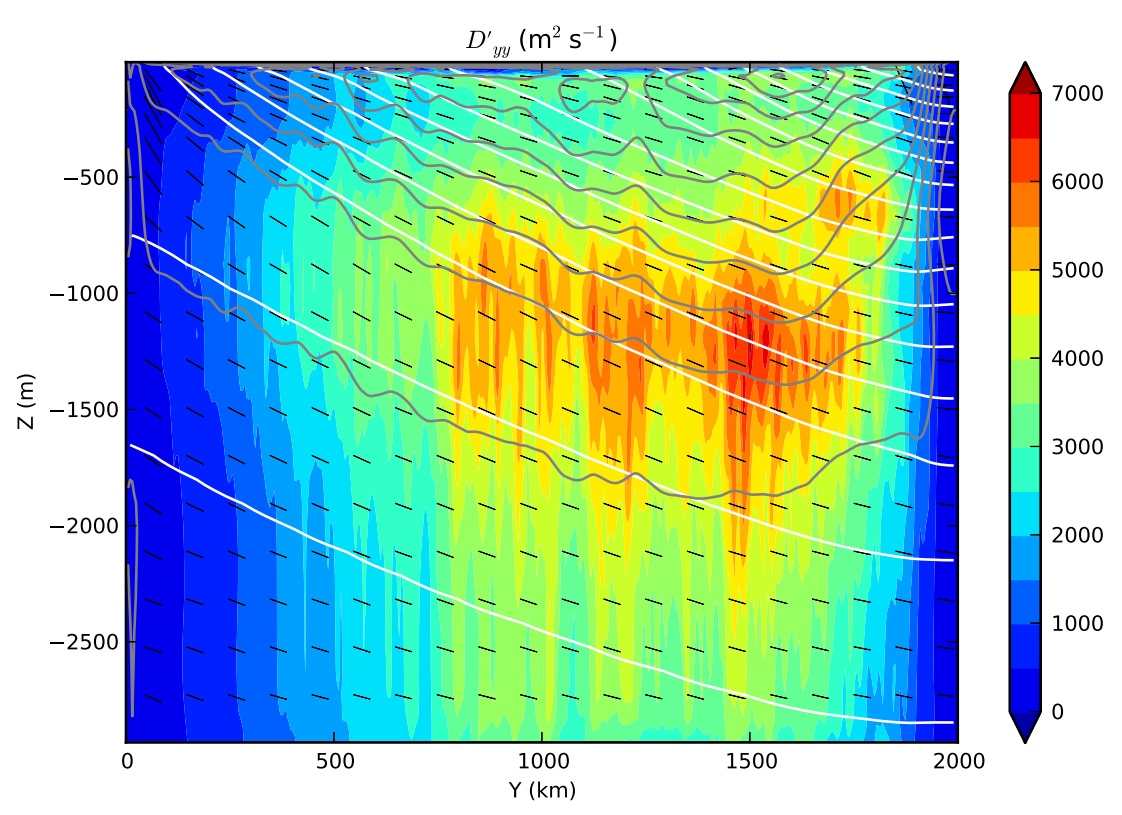

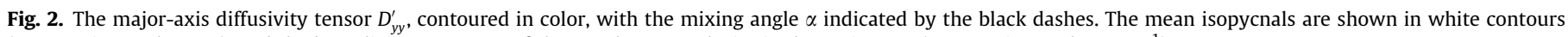
(contour interval $0.5^{\circ} \mathrm{C}$ ), and the baroclinc component of the zonal-mean velocity is shown in grey (contour interval $1 \mathrm{~cm} \mathrm{~s}^{-1}$ ). 
transformed-Eulerian-mean eddy-induced circulation and the eddy-driven "bolus transport" in isopycnal thickness-weighted averaging (PM87; McIntosh and McDougall, 1996). A complete discussion and comparison of these different conventions for defining eddy-induced advection is beyond the scope of this paper, which is focused on isopycnal mixing. Here we simply note that $\chi$ is indeed quite close to the eddy-induced transport $\Psi^{*}$ diagnosed by Abernathey et al. (2011), calculated as $\Psi_{\text {iso }}$ (the thickness-weighted circulation defined in (1)) minus the Eulerian component. As seen in Fig. 3, the spatial structure and magnitude are quite close, but $\chi$ contains more small scale variance. This similarity supports the notion that the transport processes at work in our model are not heavily tracer dependent, and that the transport of passive tracers, buoyancy, and mass can be characterized accurately by a single tensor $\mathbf{K}$.

\subsubsection{Nakamura effective diffusivity}

The framework developed by Nakamura (1996) has gained widespread use in assessing lateral mixing in the ocean and atmosphere (Nakamura and Ma, 1997; Haynes and Shuckburgh, 2000a; Haynes and Shuckburgh, 2000b; Marshall et al., 2006; Abernathey et al., 2010; Klocker et al., 2012a). This framework relies on a tracer-based coordinate system, in which the flux across tracer isosurfaces can be characterized by an effective diffusivity, which depends only on the instantaneous tracer geometry. A similar concept was developed by Winters and D'Asaro (1996).

The effective diffusivity is defined as

$K_{\text {eff }}=\kappa \frac{L_{e}^{2}}{L_{\min }^{2}}$,

where $L_{e}$ is the equivalent length of a tracer contour that has been stretched by eddy stirring and $L_{\min }$ is the minimum possible length of such a contour, in this case, simply the domain width in the zonal direction. For further background and details regarding the $K_{\text {eff }}$ calculation, the reader is referred to Marshall et al. (2006).

As described in the preceding section, the model was constructed to be as adiabatic as possible, with explicit horizontal and vertical diffusion set to zero. However, the effective diffusivity framework requires a constant small-scale background horizontal diffusivity $\kappa$. Therefore, in the tracer advection for the effective diffusivity experiments, we used an explicit horizontal diffusivity of $\kappa=50 \mathrm{~m}^{2} \mathrm{~s}^{-1}$. Analysis of the tracer variance budget indicated that numerical diffusion elevated this value slightly, to $55 \mathrm{~m}^{2} \mathrm{~s}^{-1}$. We performed our experiments by initializing a passive tracer with concentration $c=y$ and allowing it to evolve under advection and diffusion for two years. Every month, a snapshot of $c$ and $T$ was output. This procedure was repeated for 10 consecutive twoyear periods, to create a smooth ensemble-average picture of the evolution of $K_{\text {eff }}$ over two years.

The 3D tracer field must be sliced into 2D surfaces in order to compute $K_{\text {eff }}(y)$. The most straightforward way to accomplish this is to examine surfaces of $c$ at constant $z$; we call this $K_{\text {eff }}^{H}$. However, since the mixing angle is along isopycnals, a more physically relevant choice is to project $c$ into isopycnal coordinates; the effective diffusivity computed from this projection we call $K_{\text {eff }}^{\text {iso }}$. Abernathey et al. (2010) tried both methods, and here we do the same.

After two months, the overall magnitude of both $K_{\text {eff }}$ calculations stabilizes and remains roughly constant, as does the spatial structure of $K_{\text {eff }}^{\text {iso }}$. The spatial structure of $K_{\text {eff }}^{H}$, on the other hand, continues to evolve over the two year period, departing further and further from $K_{\text {eff }}^{\text {iso }}$. The results of one $K_{\text {eff }}$ ensemble calculation (at 10 months) are shown in Fig. 4. Comparing this figure with Fig. 2, we see that $K_{\text {eff }}^{\text {iso }}$ is very similar in magnitude and spatial structure to $D_{y y}^{\prime}$. This agreement between these two diagnostics,
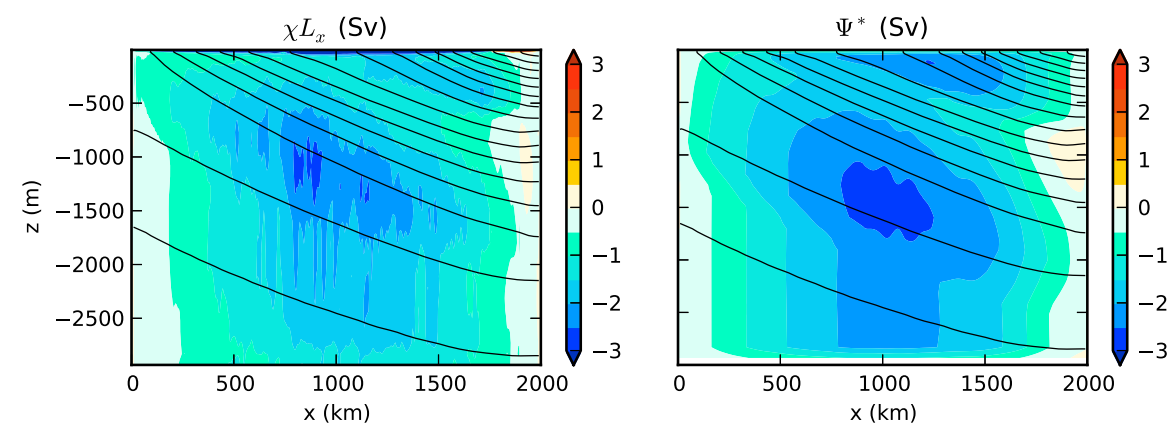

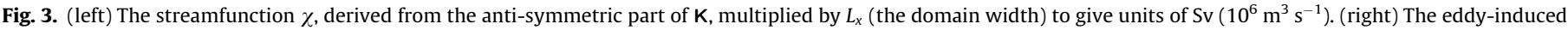
bolus transport streamfunction $\Psi^{*}$ from Abernathey et al. (2011), calculated as $\Psi_{\text {iso }}$ (the thickness-weighted circulation) minus the Eulerian component.
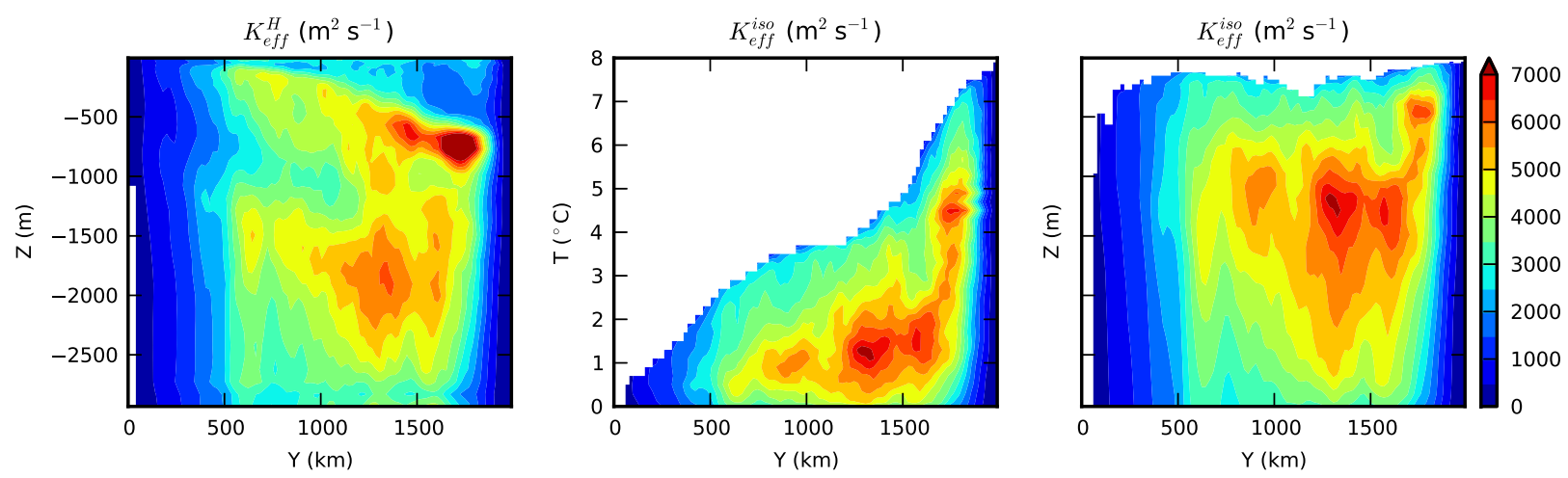

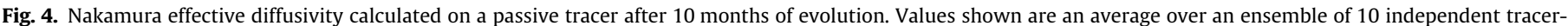

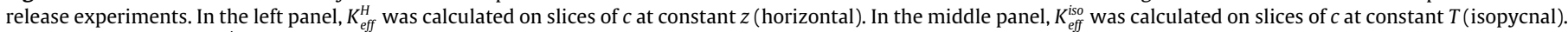
The right panel shows $K_{\text {eff }}^{\text {iso }}$ mapped back to depth space using the mean isopycnal depths. 

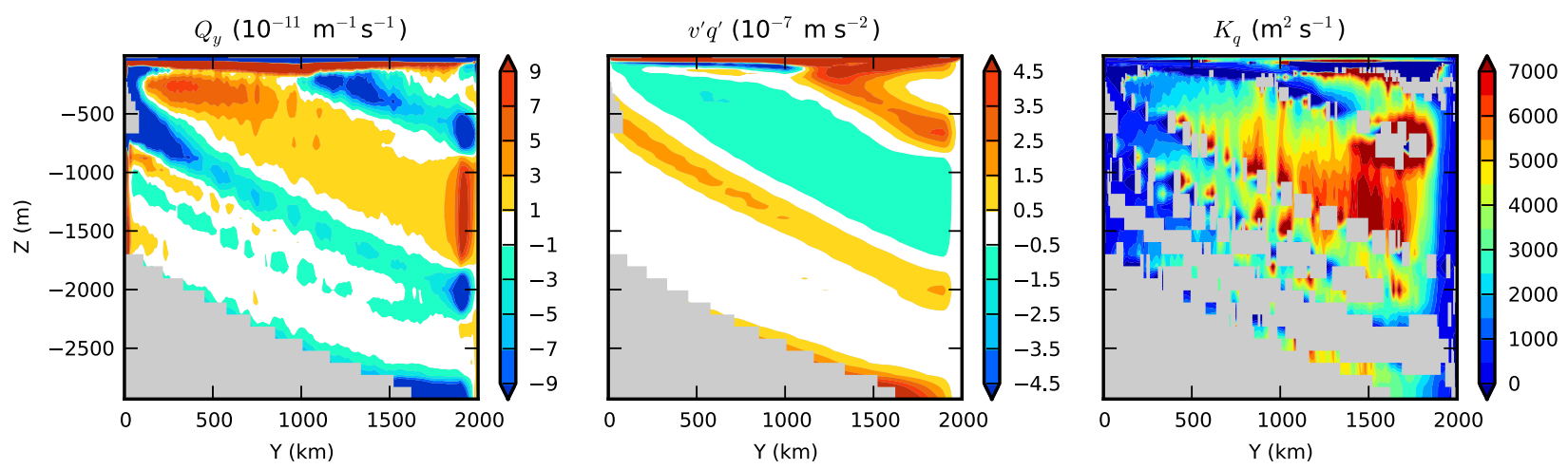

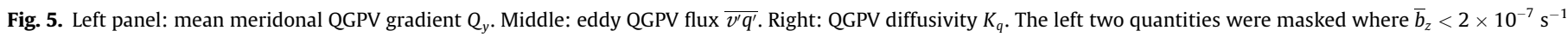

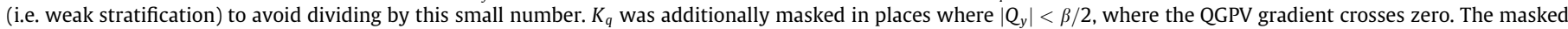
areas are colored gray.

based on quite different methods, is expected but nevertheless encouraging. $K_{\text {eff }}^{H}$, on the other hand, while having the right general magnitude, has significant differences in spatial structure. From this we conclude that $K_{\text {eff }}^{H}$ is somewhat misleading diagnostic; since the mixing angle $\alpha$ is aligned with the isopycnals, it is not physically justified to examine the tracer on level surfaces. $K_{\text {eff }}^{\text {iso }}$, on the other hand, is a robust diagnostic of isopycnal mixing.

\subsection{Active tracers}

Now we compute flux-gradient diffusivities for active tracers. By active tracers we mean scalars which are advected by the flow but which also affect the dynamics of the flow. The active tracers we consider are potential vorticity (both planetary Ertel and quasi-geostrophic varieties) and buoyancy. Also, unlike the passive tracers, these active tracers are forced at the surface, and their zonal means have reached a steady-state equilibrium. Therefore, it is interesting to ask whether they experience the same diffusivity as the passive tracers.

\subsubsection{QGPV diffusivity}

Quasi-geostrophic theory predicts that stirring by mesoscale eddies will lead to a down-gradient flux of quasi-geostophic potential vorticity (QGPV) in the ocean interior (Rhines and Young, 1982). Although this down-gradient relationship cannot be expected to hold locally at every point in the ocean, it is much more robust in a zonally-averaged context, which eliminates rotational fluxes from the enstrophy budget (Marshall and Shutts, 1981; Wilson and Williams, 2004). Although our model is based on primitive equations, certain quasi-geostrophic quantities can nevertheless be calculated (Treguier et al., 1997). Of interest here is the eddy QGPV flux ${ }^{1}$

$\overline{v^{\prime} q^{\prime}}=f_{0} \frac{\partial}{\partial z}\left(\overline{\frac{v^{\prime} b^{\prime}}{\bar{b}_{z}}}\right)$

and the background meridional QGPV gradient

$Q_{y}=\beta-f_{0} \frac{\partial s_{b}}{\partial z}$,

where $s_{b}=-(\partial \bar{b} / \partial y) /(\partial \bar{b} / \partial z)$ is the mean isopycnal slope. The QGPV diffusivity is then defined as

$K_{q}=-\overline{v^{\prime} q^{\prime}} / Q_{y}$.

\footnotetext{
1 The QGPV flux also includes a Reynolds-stress term $\partial_{y}\left(\overline{u^{\prime} v^{\prime}}\right)$. In our model, this term is an order of magnitude smaller, as expected from standard oceanographic scaling arguments (Vallis, 2006), and has therefore been neglected. Consistently, the relative vorticity gradient has also been neglected in the definition of $Q_{y}$.
}

The importance of the QGPV flux in the momentum budget is discussed in Treguier et al. (1997).

All three of these quantities are plotted in Fig. 5. First we note that, where $Q_{y}$ is nonzero, there is indeed a strong anti-correlation between $Q_{y}$ and $\overline{v^{\prime} q^{\prime}}$, supporting the notion of a down-gradient transfer of QGPV. This is reflected by the fact that $K_{q}$ is positive nearly everywhere. (The relationship breaks down near the surface, which we attribute to the presence of strong forcing terms and an unstratified mixed layer, making the QG approximation itself invalid.) Furthermore, comparing Fig. 5 with Fig. 2, we see a strong resemblance between $K_{q}$ and $D_{y y}^{\prime}$, both in magnitude and spatial structure. The calculation of $K_{q}$ involves computing many derivatives in both $y$ and $z$. We expected to find a very noisy result, and are consequently pleasantly surprised by this agreement. $K_{q}$ is also very similar to $K_{e f f}^{\text {iso }}$, supporting the choice by Abernathey et al. (2010) to equate these quantities in a diffusive closure for the eddy QGPV flux.

\subsubsection{Isopycnal planetary ertel PV diffusivity}

Through the well-known correspondence between the quasigeostrophic framework and analysis in isopycnal coordinates, the QGPV flux can be recast as a flux of Ertel potential vorticity along isopycnals (Andrews et al., 1987). Analysis of the tracer variance budget in isopycnal coordinates also supports a down-gradient diffusive closure for the PV flux in this framework (Jansen and Ferrari, 2013). Here we calculate the along-isopycnal Ertel PV diffusivity directly. In our context, the Ertel PV is very well captured by the planetary approximation, in which relative vorticity is neglected; our definition of Ertel PV is therefore $P=f \partial b / \partial z$.

The isopycnal diffusivity of Ertel potential vorticity is defined as

$K_{P}=-\overline{\hat{v} \widehat{P}^{*}} / \frac{\partial \bar{P}^{*}}{\partial y}$.

The ${ }^{-*}$ symbol indicates a generalized thickness-weighted zonal average along isopycnals, and the ${ }^{\wedge}$ symbol the anomaly from that average. (For further details of thickness-weighted averaging in isopycnal coordinates and associated notation, the reader is referred to Jansen and Ferrari (2013).) All the factors in (17) are plotted in Fig. 6, in buoyancy space rather than depth. The strong similarity between the fluxes and gradients in the QG and isopycnal frameworks confirms the mathematical correspondence between these two forms of analysis. Furthermore, the spatial structure and magnitude of $K_{P}$ in the interior is quite similar to $K_{\text {eff }}^{\text {iso }}$ (Fig. 4, middle) and, when mapped back to depth coordinates (not plotted), to $D_{y y}^{\prime}$ and $K_{q}$. The down-gradient nature of the flux also clearly breaks down in the surface layer, due to factors such as the presence of strong forcing terms and the intermittent outcropping of isopycnals. 

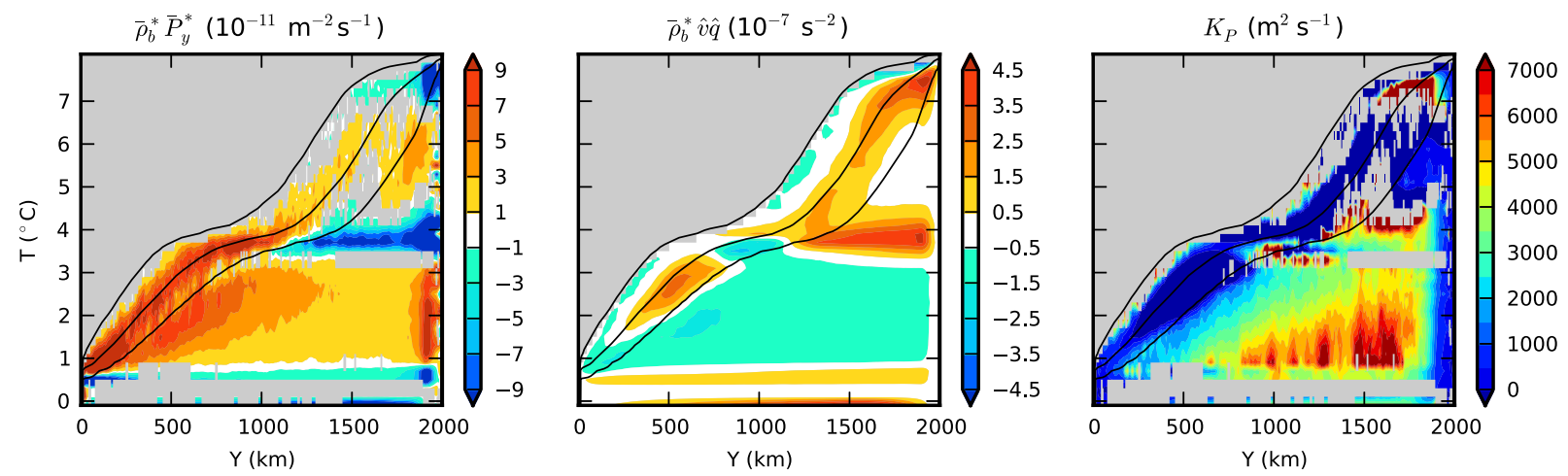

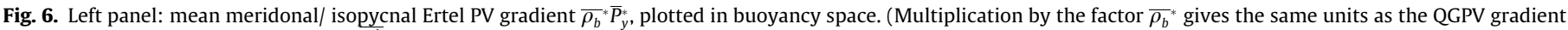

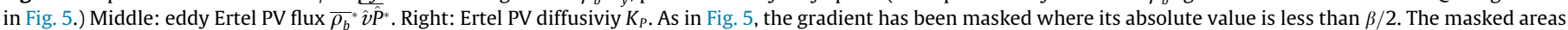
are colored gray. The black contours indicate the $5 \%, 50 \%$, and $95 \%$ levels of the surface buoyancy cumulative distribution function.

\subsubsection{Buoyancy diffusivity}

The horizontal buoyancy diffusivity is an important yet problematic quantity, defined as

$K_{b}=-\frac{\overline{v^{\prime} b^{\prime}}}{\bar{b}_{y}}$.

For quasigeostrophic, adiabatic eddies, this quantity is equal to the Gent and McWilliams (1990) transfer coefficient (Treguier et al., 1997), which plays a central role in the parameterization of eddyinduced advection in numerical models (Gent et al., 1995; Griffies, 1998) and in the theory of the Southern Ocean overturning circulation (Marshall and Radko, 2003; Nikurashin and Vallis, 2012). It is commonly also referred to as the GM coefficient or the "thickness diffusivity." The term thickness diffusivity is especially problematic when mixing rates are spatially variable; in this case it can be shown that the isopycnal thickness diffusion is not equal to $K_{b}$ and, in fact, that isopycnal thickness diffusion is more closely related to PV diffusion (see discussion in Section 3 Gent et al., 1995, of who were aware of the distinction). Nevertheless, knowledge of $K_{b}$ is a very important quantity, since nearly all numerical models use the Gent-McWilliams parameterization. In the full threedimensional case (as opposed to the zonally averaged case considered here), a different value of $K_{b}$ is defined for each of the distinct components (zonal and meridional) of the horizontal flux (Griffies, 1998).

$K_{b}$ is not, properly speaking, a diffusivity at all in the Fickian sense. This is because, in the adiabatic interior, the eddy buoyancy flux $\boldsymbol{F}_{b}$ (of which $\overline{v^{\prime} b^{\prime}}$ is only one component) is directed almost entirely perpendicular to the buoyancy gradient (Griffies, 1998; Plumb and Ferrari, 2005). There is no down-gradient eddy flux of buoyancy, only a "skew flux." In Section 3.1.1, we observed that the mixing angle $\alpha$ in the interior satisfies $\alpha \simeq-\bar{b}_{y} / \bar{b}_{z}$. This means that the contribution to $\overline{v^{\prime} b^{\prime}}$ from $-\mathbf{D} \nabla b$ is due only to the diapycnal diffusvity $D_{z z}^{\prime}$, which is negligibly small, and consequently that the eddy buoyancy fluxes are captured by $L$ alone (in fact by a single scalar $\chi$ ). Using (4) and (6), we see that

$K_{b} \simeq \chi / s_{b}$,

where $s_{b}=-\bar{b}_{y} / \bar{b}_{z}$ is the mean isopycnal slope. The buoyancy diffusivity $K_{b}$ is related to the eddy-induced streamfunction $\chi$ and the isopycnal slope, i.e. to the advective part of the eddy transport, not the diffusive part. This relation is in fact a key assumption of the Gent and McWilliams (1990) parameterization.

We have plotted both sides of (19) as well as a scatter plot of their relationship in Fig. 7, illustrating the similarity between the two quantities. (The small differences between $K_{b}$ and $\chi / s_{b}$ can be attributed to diabatic effects.) Comparison with Fig. (2) reveals significant differences between $K_{b}$ and $D_{y y}^{\prime}$. Noting the different color scales used in Figs. 7 and 2, it is evident that overall magnitude of $K_{b}$ is roughly half that of $D_{y y}^{\prime}$. Significant differences in spatial structure are also present. For instance, $K_{b}$ has its highest values at the bottom and top of the water column, while $D_{y y}^{\prime}$ has its maximum at mid-depth. It is particularly important to point out these differences because it is quite common to assume that $D_{y y}^{\prime}=K_{b}$ in the context of eddy parameterization (Gent and McWilliams, 1990; Gent et al., 1995; Griffies, 1998). Such an assumption is clearly not supported by our simulations. Similarly, Liu et al. (2012) used an adjoint-based method to infer $K_{b}$ and then discussed the results in terms of the mixing-length ideas of Ferrari and Nikurashin (2010), which are more relevant to $D_{y y}^{\prime}$ of $K_{e f f}^{\text {iso }}$. Our results, in addition to those of previous authors (SM09;
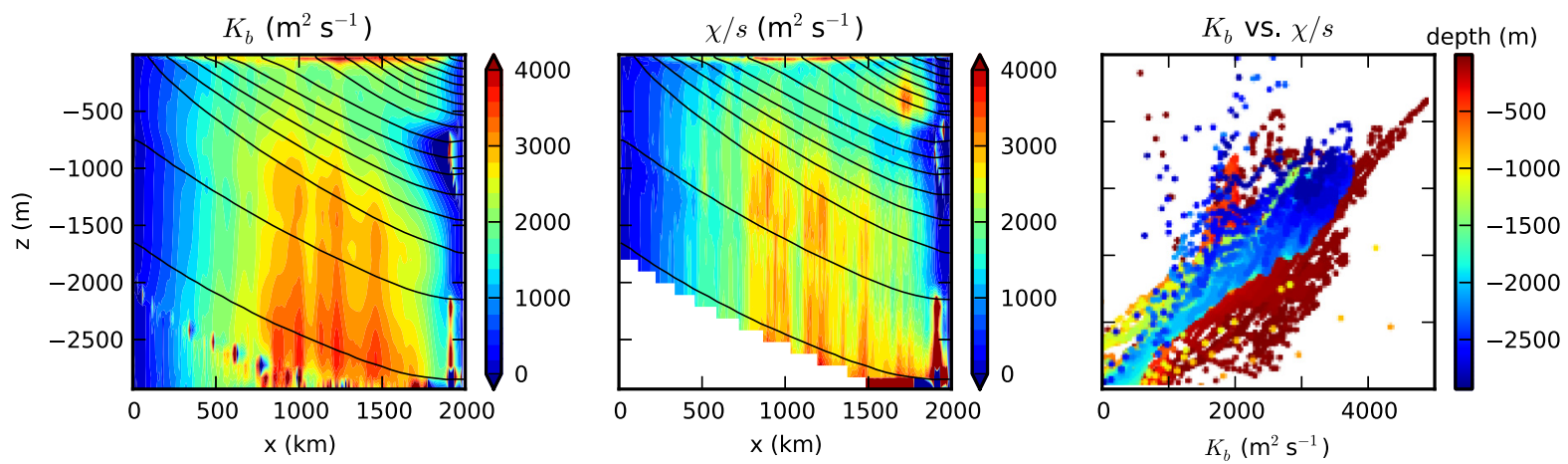

Fig. 7. (left) horizontal buoyancy diffusivity $K_{b}$ calculated from (18). (center) $\chi / s_{b}$. (right) Scatter plot of the two quantities. 

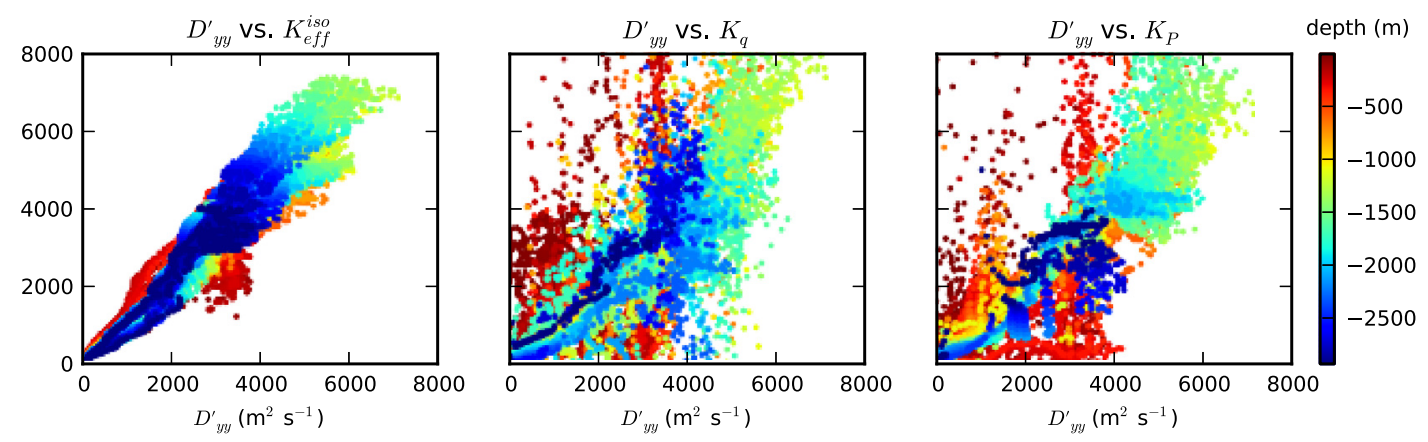

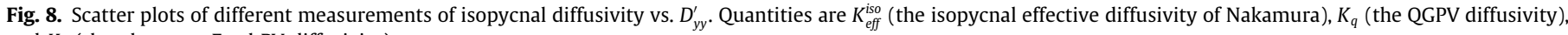
and $K_{P}$ (the planetary Ertel PV diffusivity).

Treguier, 1999) suggest this comparison is unsound. In Section 5 , we will further explore the relationship between $K_{b}$ and $D_{y y}^{\prime}$ and discuss the parameterization problem.

\subsection{Summary}

So far in this section we have seen strong agreement between different perfect diagnostics of isopycnal mixing. In particular, $D_{y y}^{\prime}, K_{e f f}^{i s o}, K_{q}$, and $K_{P}$ all give a similar picture of along-isopycnal mixing rates. The strength of along-isopycnal mixing varies between 3000 and $7000 \mathrm{~m}^{2} \mathrm{~s}^{-1}$ in the middle of the domain, with a pronounced peak between 1000 and $1500 \mathrm{~m}$ depth. Mixing rates fall off sharply at the northern and southern edges of the domain. To make the comparison between diagnostics more quantitative, we now show scatter plots of $K_{e f f}^{i s o}, K_{q}$, and $K_{P}$ vs. $D_{y y}^{\prime}$ (which we take to be our reference "truth" for isopyncal mixing rates) in Fig. 8. To construct these plots, the diffusivities defined in isopycnal coordinates were first interpolated to depth space. The figure reveals a tight relationship between $D_{y y}^{\prime}, K_{e f f}^{\text {iso }}$, which are both well-defined everywhere in the domain. The comparison with the PV diffusivities is more noisy, especially for shallow depths, where QG scaling breaks down due to the presence of the mixed layer and the presence of forcing (including strong diapycnal mixing) disrupts the simple down-gradient diffusion of PV. The spread is also due to the fact that the PV gradient goes through zero several times in the domain, at which points the diffusivity definition breaks down. Nevertheless, for depths below $500 \mathrm{~m}$, the correlation of $K_{q}$ and $K_{P}$ with $D_{y y}^{\prime}$ is good. The figure also reveals, from a different perspective, that the highest diffusivities for all four quantities occur at depths between 1000 and $1500 \mathrm{~m}$.

As expected from previous studies (SM09; Treguier, 1999), the buoyancy diffusivity $K_{b}$ does not agree with the other mixing diagnostics, differing both in magnitude and vertical structure. This reflects the fact that $K_{b}$ is a "skew" diffusivity rather than an isopycnal diffusivity (Griffies, 1998). We now turn to the question of how, and how accurately, the isopycnal mixing rates can be inferred from experiments in the field.

\section{Practical mixing diagnostics}

\subsection{Lagrangian diffusivity}

One of the two most common methods to estimate isopycnal diffusion in observational programs is the use of Lagrangian trajectories of either surface drifters or subsurface floats (e.g. Davis, 1991; LaCasce, 2008). (The other method, described in the next subsection, is to use tracer release experiments.) Lagrangian diffusivities are calculated from the mean square separation of an ensemble of $N$ drifters or floats (called simply "particles" from here on) from their starting positions. This is the single-particle diffusivity of Taylor (1921):

$K_{1 y}\left(y_{0}, t\right)=\frac{1}{2} \frac{d}{d t}\left[\frac{1}{N} \sum_{i=1}^{N}\left(y_{i}(t)-y_{i 0}\right)^{2}\right]$.

Here $y_{i}(t)$ is the meridional position of a particle released at $y_{i 0}$ at $t=0$. Lagrangian diffusivities can also be calculated using the mean-square separation of particles relative to each other. Both the single-particle diffusivity and the relative diffusivity asymptote at long times (e.g. Davis, 1985). As shown by Taylor (1921), these eddy diffusivities are equal to the integral of the Lagrangian autocorrelation function, which in case of the single-particle diffusivity can be written as:

$K_{1 y}\left(y_{0}, t\right)=\int_{0}^{t} R_{v v}\left(y_{0}, \tau\right)$

where

$R_{v v}\left(y_{0}, \tau\right)=\frac{1}{N} \sum_{i=1}^{N} v_{i}(\tau) v_{i}(0)$

Here $v_{i}(t)$ is the meridional velocity of particle $i$. If the autocorrelation reaches zero after a finite time, the Lagrangian diffusivity $K_{1 y}\left(y_{0}, t\right)$ will asymptote to a constant value (Taylor, 1921).

Here it is important to note that it is necessary to have sufficient Lagrangian statistics to resolve this Lagrangian autocorrelation function until it decorrelates; the error is expected to decrease as $n^{-1 / 2}$, where $n$ is the number of particles (Davis, 1994). Klocker et al. (2012b) have shown that this Lagrangian autocorrelation function has two parts-an exponential decaying part and an oscillatory part. If integrating just over the exponential decaying part, one would derive an eddy diffusivity for the case in which the mean flow does not influence the diffusivity. But as shown by several recent studies (Marshall et al., 2006; Abernathey et al., 2010; Ferrari and Nikurashin, 2010), eddy diffusivities are influenced by the mean flow; this can be seen as the oscillatory part of the Lagrangian autocorrelation function (Klocker et al., 2012b). Resolving this oscillatory part requires a much larger number of particles, and therefore leads to strong limitations in observational programs due to the limited number of drifters and floats deployed in those programs. (See Klocker et al. (2012b) for a more detailed exploration of the issue of using limited Lagrangian statistics to derive eddy diffusivities in observational studies.).

In numerical simulations, we can just increase the number of floats until the errors are vanishingly small. To calculate eddy diffusivities in this study, floats are deployed at every grid point (i.e. every $5 \mathrm{~km}$ ) within a region which extends over the whole model domain in the zonal direction and over a width of $100 \mathrm{~km}$, centered in the channel, in meridional direction. This results in a total of 4000 floats at each depth. In the vertical, there were 40 different 
release depths corresponding to the model's vertical grid. The floats are then advected for one year by the full three-dimensional flow, with positions output every day. Lagrangian eddy diffusivities are calculated at each depth according to (21), with the eddy diffusivity being calculated as an average over days 30-40. Examples for the Lagrangian autocorrelation function, $R_{v v}$, and the Lagrangian eddy diffusivity, $K_{1 y}$ are shown in Fig. 9a for floats deplayed at a depth of $100 \mathrm{~m}$ and $9 \mathrm{~b}$ for floats deployed at a depth of $1500 \mathrm{~m}$. Fig. 9a shows a typical example for a depth where the mean flow plays an important role in suppressing eddy diffusivities, with $R_{v v}$ showing an exponential decay and an oscillatory part, leading to an eddy diffusivity $K_{1 y}$ which first increases to approx. $8000 \mathrm{~m}^{2} \mathrm{~s}^{-1}$ and then converges at approx. $4000 \mathrm{~m}^{2} \mathrm{~s}^{-1}$. Fig. $9 \mathrm{~b}$ shows both $R_{v v}$ and $K_{1 y}$ for a depth where the mean flow does not play an important role, i.e. $R_{v v}$ only shows an exponential decay and $K_{1 y}$ increases until converging at approx. $3700 \mathrm{~m}^{2} \mathrm{~s}^{-1}$. In both cases the Lagrangian autocorrelation function decorrelates after approx. 30 days. The vertical profile of Lagrangian diffusivities is shown in Fig. 13 (the overall comparison figure, discussed subsequently) and agrees well with other estimates of eddy diffusivities.

\subsection{Tracer release}

Another possible method to measure isopycnal diffusion in the ocean is through the use of deliberate tracer release experiments. Such techniques have already been successfully employed to estimate diapycnal mixing by Ledwell and collaborators (Ledwell and Bratkovich, 1995; Ledwell et al., 1998; Ledwell et al., 2011). In these experiments, a passive dye is released as close as technically possible to a target isopycnal in the ocean and its subsequent evolution monitored over a few years. To quantify the vertical diffusion, the tracer field is first averaged isopycnaly into one vertical
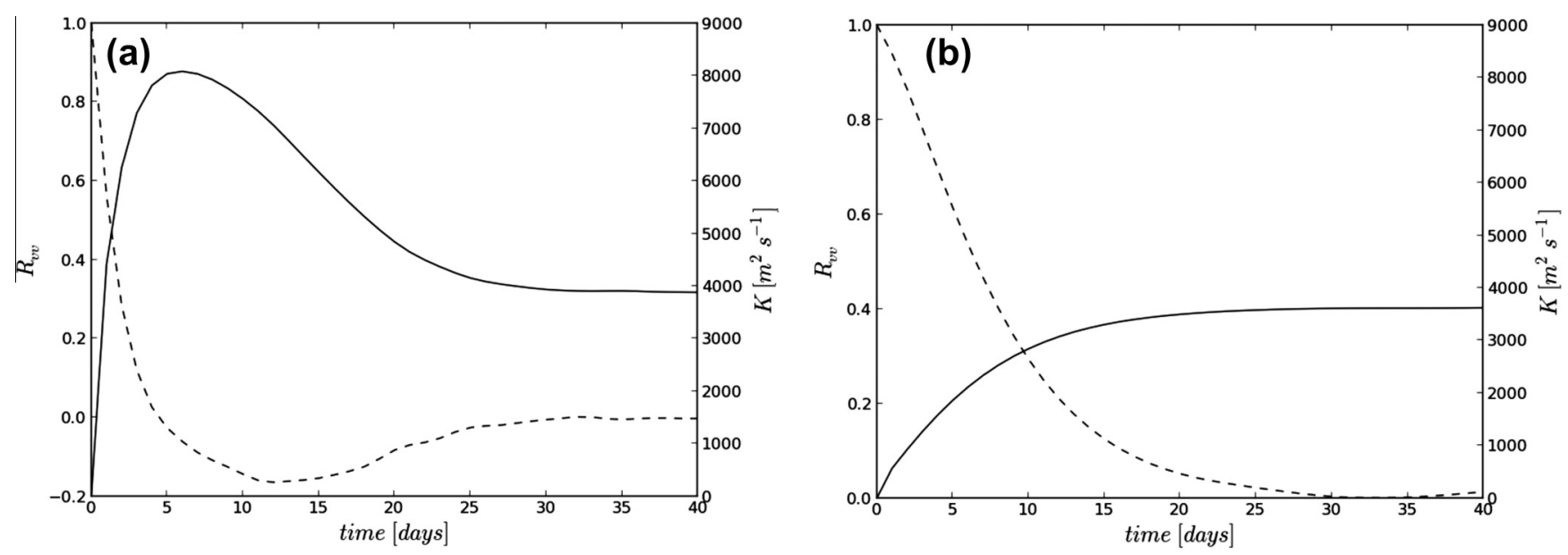

Fig. 9. Lagrangian autocorrelation function $R_{v v}$ (dashed) and $K_{1 y}$ (solid) from the particle release experiments at depths of (a) $100 \mathrm{~m}$ and (b) $1500 \mathrm{~m}$.

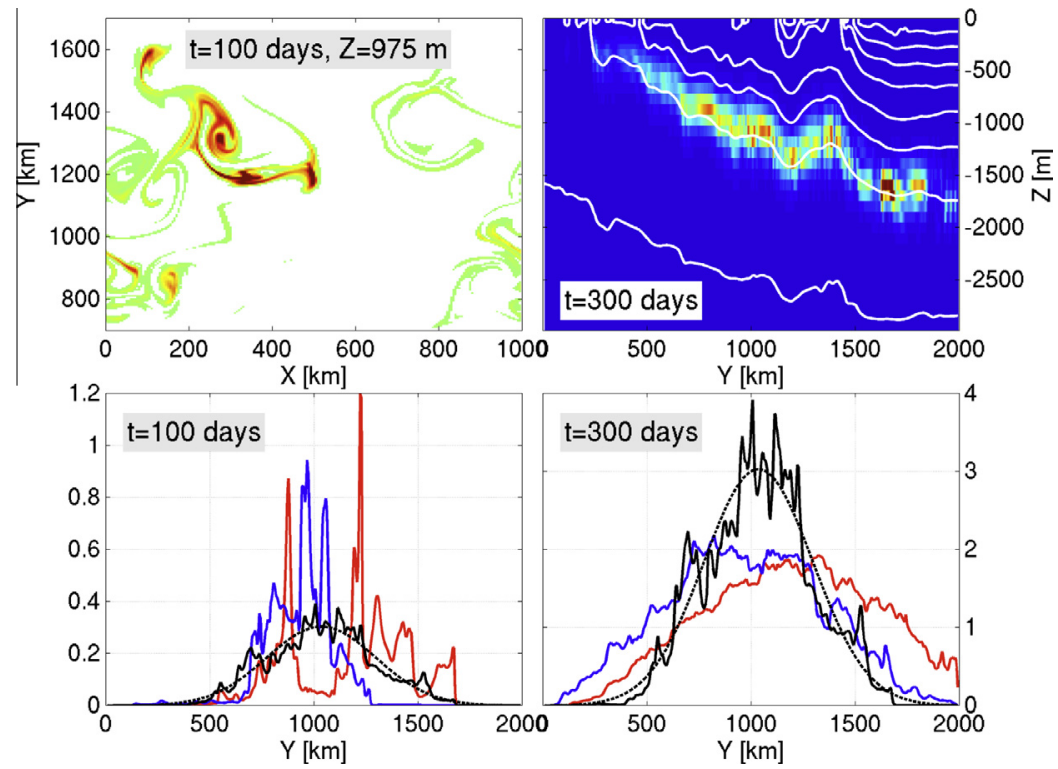

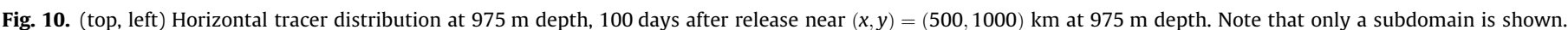

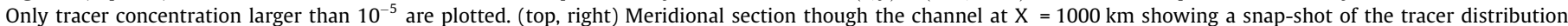

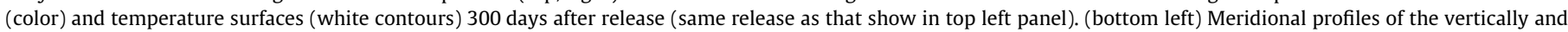

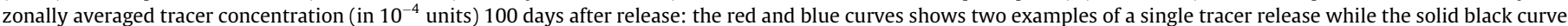

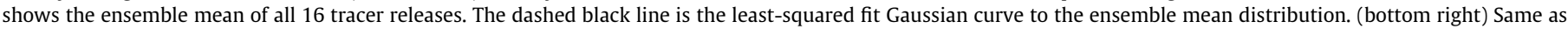

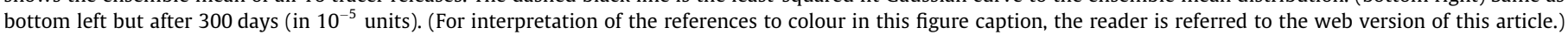


profile. These profiles are well approximated by a Gaussian whose width $\sigma$ evolves linearly with time (as expected if the tracer field spread vertically according to a simple one dimensional diffusion equation). The vertical diffusion $\kappa_{v}$ is then given by $\kappa_{v}=(1 / 2) d \sigma / d t$. This method was also successfully applied to the estimation of the effective diapycnal diffusion in a numerical model in a setup very similar to the one used here (Hill et al., 2012).

One hopes that isopycnal diffusion in the ocean could be estimated using similar techniques by taking advantage of already collected data (e.g. from the NATRE and DIMES campaigns; Ledwell et al., 1998; Gille et al., 2012). To achieve this, one could monitor the isopycnal spreading of the tracer by summing its 3D distribution vertically. To simplify further the problem here, we will zonally average the resulting $2 \mathrm{D}$ map into a $1 \mathrm{D}$ profile and focus on the meridional diffusivity $K_{I}$. Unfortunately, one can readily see that the tracer distribution is very patchy and its meridional profile is poorly approximated by a Gaussian. Fig. 10 illustrates this point in the channel, plotting the tracer distribution 100 days after release. (Details of the tracer-release experiments and diagnostic methods are given in Appendix B.) The tracer patch is stretched into long narrow filaments, cascading to small scales. Such behavior is also observed in the real ocean (see Fig. 18 from Ledwell et al. (1998)). Unlike the diapycnal case, the isopycnal dispersion of a tracer patch does not fit a one-dimensional diffusion equation, at least initially, effectively preventing a reliable estimation of $K_{I}$.

One possible way to circumvent this issue is to consider an ensemble of tracer releases. One expects that in an average sense, the tracer does behave in a diffusive way. To test this, we perform 16 tracer releases in the model: 8 tracers are released simultaneously $125 \mathrm{~km}$ apart along the center of the channel, followed by a second set of 8 releases 300 days later. The ensemble-mean profiles at 100 and 300 days after release are shown in Fig. 10 (bottom, black solid). Contrary to profiles from single releases, the ensemble-mean profile already approaches a Gaussian shape after 100 days. Importantly, the width of the best-fit Gaussian curve to the ensemble-mean profile (dashed black) grows linearly with time after 150 days at most depths (see Appendix B for details).

The isopycnal diffusivity in the channel, estimated from the 16member ensemble mean, is plotted as a function of depth in

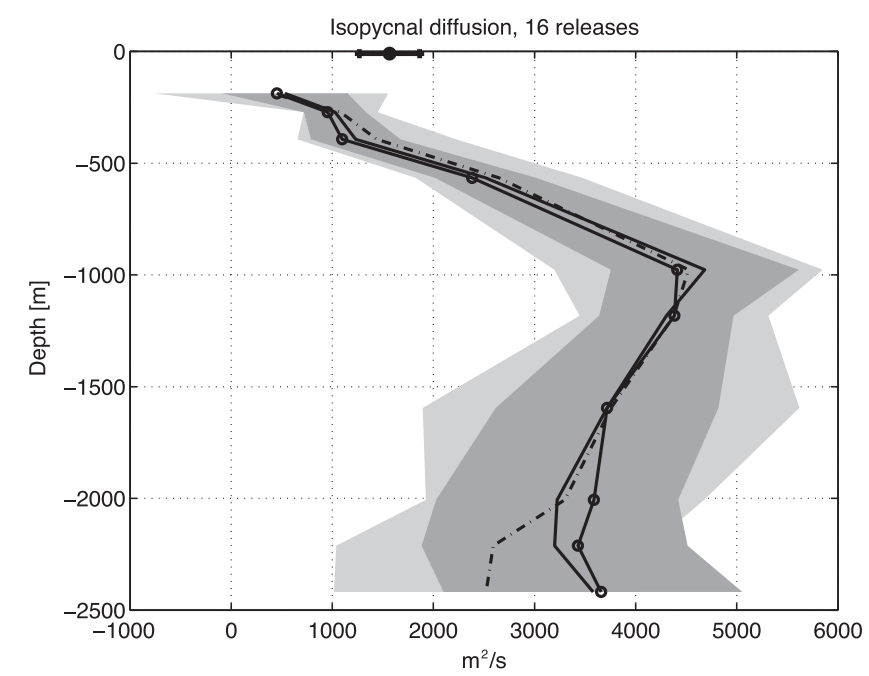

Fig. 11. Vertical profiles of the isopycnal diffusivity $K_{I}$ estimated from tracer release experiments in the channel. The thick line denotes values estimated from monitoring the evolution of the 16-member ensemble-mean tracer at each depth. The mean ( \pm one standard deviation) of isopycnal diffusivities computed by following each tracer individually (16 values) are shown by a dashed-dotted line and light grey shading. Similar quantities from 2-member ensemble-mean are shown in solid black and dark grey shading.
Fig. 11. It increases from about $500 \mathrm{~m}^{2} \mathrm{~s}^{-1}$ in subsurface to slightly more than $4000 \mathrm{~m}^{2} \mathrm{~s}^{-1}$ around $1100 \mathrm{~m}$ depth, and then decreases to $3500 \mathrm{~m}^{2} \mathrm{~s}^{-1}$ near the bottom. Note that subsurface (300$400 \mathrm{~m}$ ) values are likely underestimates because, at these depths, the tracers rapidly spread along isopycnals up to the surface diabatic layer and then horizontally at the surface (see details in Appendix B). To obtain a more robust estimate near the surface, a set of 16 tracer patches were released right into the mixed layer, leading to an estimation of a surface (horizontal) diffusivity of about $1500 \mathrm{~m}^{2} \mathrm{~s}^{-1}$; a slightly higher value than in subsurface which is more consistent with the other estimates.

To give a sense of the uncertainties, the diffusivities estimated from single tracer releases were also computed. The mean plusor-minus one standard deviation of those 16 estimates (at each depth) are shown with a dashed black line and a light grey shading. Similarly, diffusivities from pairs of tracer releases were also computed (shown in dark grey shading and solid line). Uncertainties associated with a single tracer release range from pm500 $\mathrm{m}^{2} \mathrm{~s}^{-1}$ near $500 \mathrm{~m}$ depth to $\pm 1000 \mathrm{~m}^{2} \mathrm{~s}^{-1}$ or more below a $1000 \mathrm{~m}$. It appears that estimates between 500 and $1000 \mathrm{~m}$ deep would be somewhat robust. However, our results suggest that detection of a peak of mixing in the water column would be very difficult from single tracer releases at a few selected depths.

\section{Comparison of all diagnostics}

\subsection{Averaging method}

In Section 3 we saw that many of the different perfect diagnostics $\left(D_{y y}^{\prime}, K_{e f f}^{\text {iso }}, K_{q}\right.$ and $\left.K_{P}\right)$ give similar results. Now we compare these results with the practical diagnostics discussed above. The central obstacle in this comparison is the question of how to average meridionally the perfect diagnostics, which are functions of $y$ and $z$, to compare with the practical diagnostics, which are just functions of $z$. The tracers and particles for the practical experiments were released at the center of the domain and spread meridionally along isopycnals for up to 300 days before encountering the boundaries. This results in a single value of diffusivity for each release depth, or equivalently, release isopycnal. ${ }^{2}$ But as the particles/ tracers experience spread away from the center of the channel, they experience weaker mixing towards the sides of the domain.

Our procedure is to average the perfect diagnostics in isopycnal bands of thickness $\Delta T$ over a meridional extent $\Delta y$, centered on the middle of the channel. Formally this average can be expressed as

$\langle K\rangle\left(T_{0}\right)=\frac{1}{A} \int_{L_{y}-\Delta y / 2}^{L_{y}+\Delta y / 2} \int_{T(z)=T_{0}-\Delta T / 2}^{T(z)=T_{0}+\Delta T / 2} K d y d z$,

where $T_{0}$ is the target isopycnal and $A$ is the cross-sectional area over which the integral is performed. ${ }^{3} \Delta T$ effectively sets the vertical resolution of the averaged quantity, while $\Delta y$ controls the width over which it samples. Larger $\Delta y$ are associated with smaller $\langle K\rangle$, since the diffusivities tend to fall off away from the center of the channel. This effect is illustrated in Fig. 12, which shows $\left\langle D_{y y}^{\prime}\right\rangle$ for different values of $\Delta y$. The figure also shows the difference between isopycnal averaging and simple horizontal averaging (i.e. averaging at constant $z$ ), which is a more straightforward way to produce depth

\footnotetext{
${ }^{2}$ It would be possible in principle to calculate the practical diagnostics also as functions of $y$. But, in the spirit of simulating field experiments, we do not explore this possibility as it involves an even greater number of releases.

${ }^{3}$ Nakamura (2008) suggests that the proper way to average a spatially variable diffusivity is through a harmonic mean. We tested this, however, and found it to produce spurious results. This is because the harmonic mean is very sensitive to the presence of small values. Since our diffusivities are calculated numerically and contain some degree of noise at the grid scale, isolated small values can greatly influence the harmonic mean. For this reason, we prefer the simple arithmetic mean.
} 

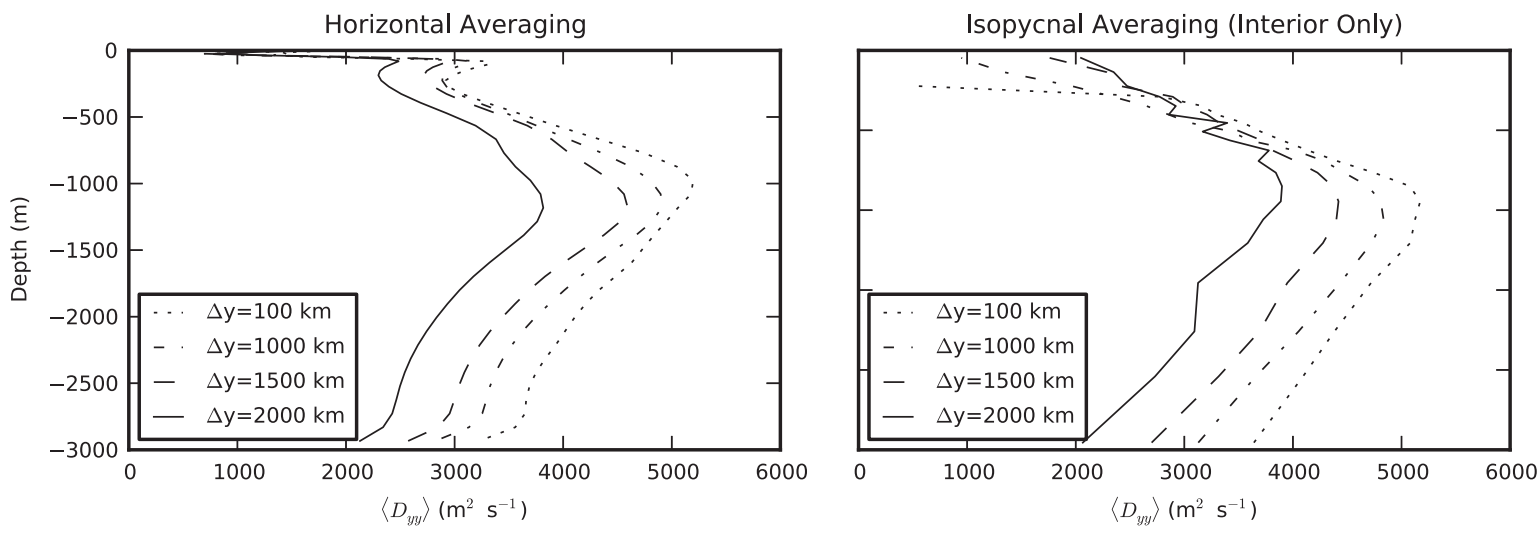

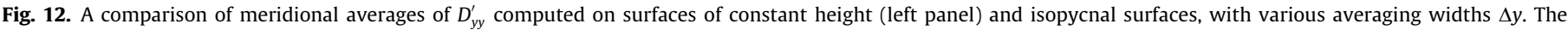
average at constant height includes the whole domain, while the isopycnal average excludes the surface diabatic layer.

profiles but is physically unsound. Instead, we map our profiles of $\langle K\rangle(T)$ to depth coordinates using the temperature profile $T(z)$ at the tracer and particle release latitude in the center of the domain. ${ }^{4}$

To fairly compare our diagnostics in the interior, we must exclude the surface diabatic layer from our average. This is because PV is not diffused down gradient in the surface layer due to the presence of strong forcing, which causes $K_{P}$ to acquire negative values there (see Fig. 6). For this reason, we limit our isopycnally averaged diffusivities to the interior, which we define as the region below the isopycnal representing the $95 \%$ contour of the surface buoyancy cumulative distribution function. The effect of excluding the surface layer can be seen in Fig. 12; the horizontal average, which includes the surface layer, shows a secondary peak near the surface, while the interior-only isopycnal average does not.

The choice of $\Delta y$ clearly affects the magnitude of our averaged perfect diagnostics. We have concluded that the optimum choice is $\Delta y=1500 \mathrm{~km}$, i.e. an average over the most of the domain, excluding the area closest to the walls. This choice produced the best agreement between perfect and practical diagnostics. It is also physically consistent with the fact that the particles and tracers from the practical experiments spread out approximately over this center portion of the channel (see Fig. 10).

\subsection{Vertical profile in the interior}

The values of $\left\langle D_{y y}^{\prime}\right\rangle,\left\langle K_{\text {eff }}^{\text {iso }}\right\rangle,\left\langle K_{P}\right\rangle$ and $\left\langle K_{b}\right\rangle$ with $\Delta y=1500 \mathrm{~km}$ are all plotted in Fig. 13. ( $K_{q}$ was not plotted because it is quite sparse and noisy in the deep ocean; however, it is very similar to $K_{P}$.) Also plotted are $K_{1 y}$ from the Lagrangian experiment and $K_{I}$ from the tracer experiment. There is fairly good agreement between the diagnostics, excluding $K_{b}$. In particular, $\left\langle D_{y y}^{\prime}\right\rangle,\left\langle K_{\text {eff }}\right\rangle$, and $K_{1 y}$ show very similar magnitudes and vertical structure, with a distinct peak near $1000 \mathrm{~m}$ depth of approx. $4000 \mathrm{~m}^{2} \mathrm{~s}^{-1}$. $\left\langle K_{P}\right\rangle$ is qualitatively similar, with a sharp peak near the same depth, but its magnitude

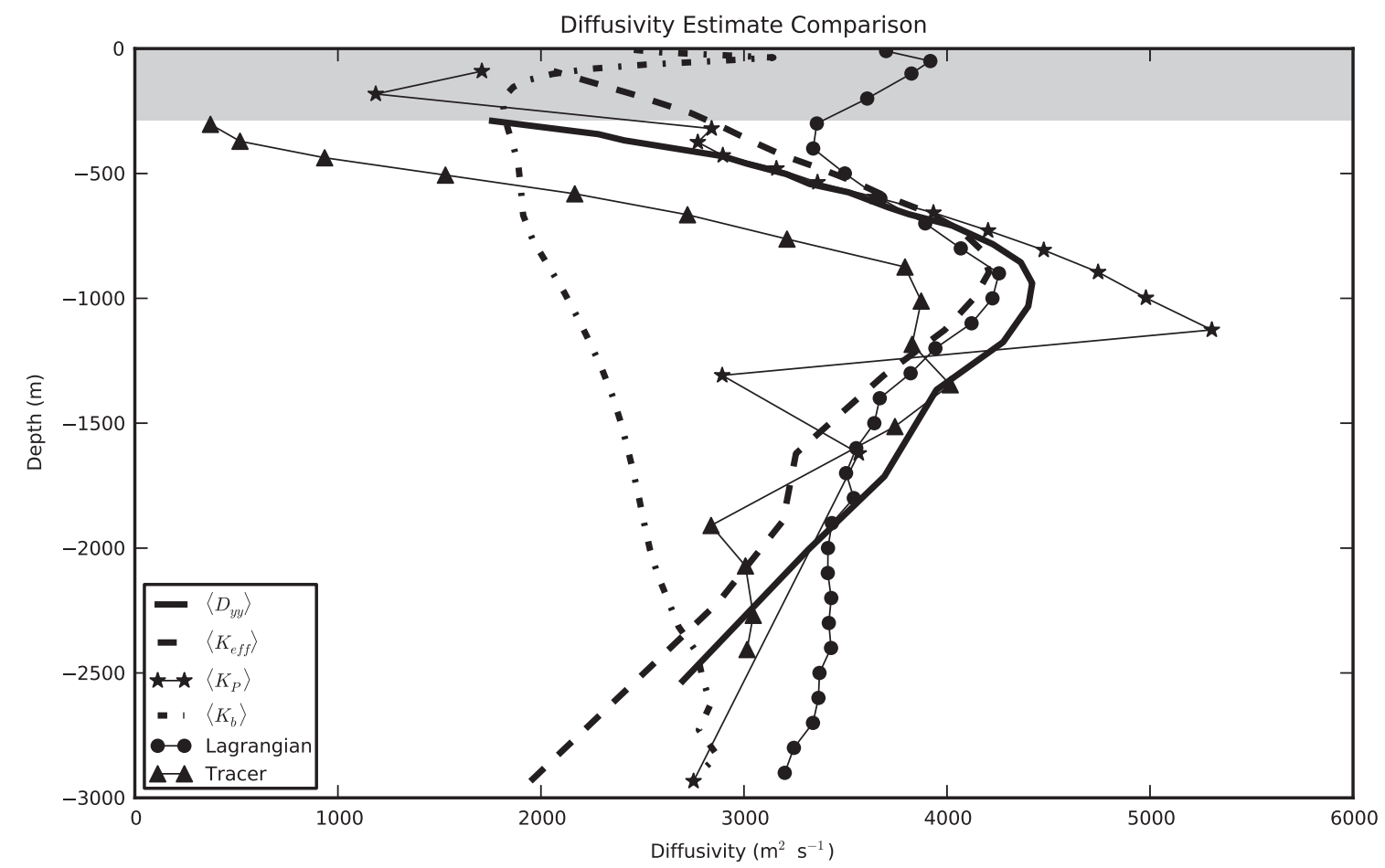

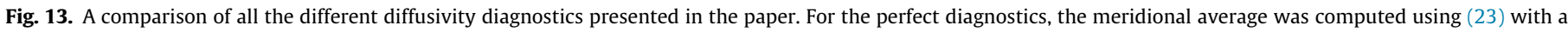
width of $\Delta y=1500 \mathrm{~m}$, and only in the interior (outside the surface diabatic layer). The average depth of the surface layer ( $280 \mathrm{~m}$ ) is indicated by the gray shaded area. 
at the peak $\left(5000 \mathrm{~m}^{2} \mathrm{~s}^{-1}\right)$ is greater. Then it drops off steeply below this peak. ( $K_{P}$ is poorly resolved below $1000 \mathrm{~m}$ because it is computed in isopycnal space; the deep is very weakly stratified, and thus there are few layers defined there.) The profile of $K_{I}$ shows a similar qualitative structure, but a slightly reduced magnitude above $1000 \mathrm{~m}$ compared with the other diagnostics. In general, there is more spread between diagnostics in the deep ocean. The overall impression from this comparison is that, despite the wide range of diagnostic methods and the ambiguities associated with the averaging process, all these diagnostics are capturing the same physical process of along-isopycnal mixing in the interior.

The vertical profile of $K_{b}$ is clearly different from the other quantities. As discussed clearly in SM09, the diffusivities of buoyancy and potential vorticity cannot be the same when $\beta$ is significant, and when there is vertical variation in the diffusivity profile (see Section 5.4 and (24) below). Nevertheless, the assumption that these two quantities are equal continues to be made in eddy parameterization schemes (for example Eden, 2010). Our results essentially confirm the conclusions of SM09, who used a doublyperiodic QG model, in a primitive-equation model with realistic meridional variations in stratification and residual circulation. In particular, our Fig. 13 agrees well with their Fig. 12. While the tracer, particle, and PV diffusivities all have a mid-depth peak, $K_{b}$ does not; instead it varies only weakly in the vertical. Its magnitude is less than half that of $K_{P}$ at the peak.

Since the perfect diagnostics were averaged only in the interior, they do not show a secondary peak near the surface. This secondary peak is clearly visible in $K_{1 y}$, the particle diffusivity. The average depth of the surface diabatic layer is also shown in Fig. 13. The secondary peak in $K_{1 y}$ clearly occurs within this surface layer. Since the surface is dynamically quite different from the interior, we now focus on the surface specifically.

\subsection{Comparison at the surface}

Near the surface, eddies transition from isopycnal mixing to horizontal mixing across the surface buoyancy gradient (Treguier et al., 1997). This transition is visible in Fig. 2, which shows that the mixing angle becoming flatter near the surface and no longer aligns with the isopycnals. In Fig. 14 , we plot $D_{y y}^{\prime}, K_{e f f}^{H}$ and $K_{b}$ all at $50 \mathrm{~m}$ depth, near the base of the mixed layer. Also plotted is a single point representing $K_{1 y}$. At the surface, we do indeed find better agreement between $K_{b}$ and the other diagnostics. This is because the near-surface eddy buoyancy flux is truly down-gradient, as opposed to the interior where it is purely skew. Nevertheless, discrepancies remain, particularly near $Y=1500 \mathrm{~km}$. We speculate that this is due to the differences in forcing and small-scale

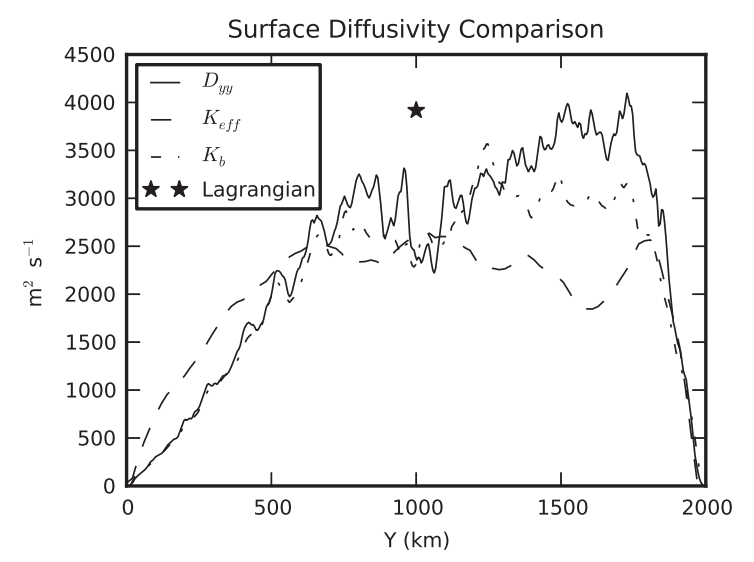

Fig. 14. A comparison of $D_{y y}^{\prime}, K_{\text {eff }}$ and $K_{b}$ at $50 \mathrm{~m}$ depth. diffusivity among the three tracers. The tracer used to calculate $K_{\text {eff }}$ was modeled with an explicit small-scale horizontal diffusion, while the others were not. Furthermore, the buoyancy is subject to an air-sea flux, which can strongly modulate the diffusivity. We have not attempted to quantify this effect here, but an in-depth treatment of the problem can be found in Shuckburgh et al. (2011).

\subsection{Relation between isopyncal diffusivity and Gent-McWilliams coefficient}

In preceding sections, we showed good agreement between all diagnostics of isopycnal mixing except for $K_{b}$, a.k.a. the skew diffusivity of buoyancy, a.k.a. the Gent-McWilliams coefficient. This would appear to be discouraging for the purposes of eddy parameterization, since most coarse-resolution models use some form of the Gent and McWilliams (1990) closure, rather than one based on potential vorticity, to represent the eddy-induced advection. The dissimilarity between $D_{y y}^{\prime}$, i.e. the true isopycnal mixing rate, and $K_{b}$, means that field experiments which aim to measure isopycnal mixing will not yield a value that can be used as a Gent-McWilliams coefficient. However, the situation is not hopeless. Quasigeostrophic theory makes a prediction for the relationship between these two quantities.

Simply using the definitions (14), (15), and (18), we can derive the following relationship between $K_{q}$ and $K_{b}$ :

$\frac{\partial}{\partial z}\left(K_{b} s_{b}\right)=K_{q}\left(\frac{\partial s_{b}}{\partial z}-\frac{\beta}{f}\right)$

(SM09). Note that this quantity has units $\mathrm{m} \mathrm{s}^{-1}$ and is equivalent to the [negative] QG-TEM eddy-induced velocity (see Treguier et al., 1997). Only if $\beta$ is negligible and $\partial K_{b} / \partial z=0$ does $K_{q}=K_{b}$.

Eq. (24) is satisfied by definition for $K_{b}$ and $K_{q}$. However, noting the similarity between $K_{q}$ and $D_{y y}^{\prime}$, we can ask whether it is also satisfied if we replace $K_{q}$ with $D_{y y}^{\prime}$ on the RHS. Such a comparison is made in Fig. 15. This figure also illustrates the error produced by assuming $K_{b}=D_{y y}^{\prime}$ (i.e. neglecting the importance of the vertical structure) and by neglecting $\beta$. We can see that using $D_{y y}^{\prime}$ in place of $K_{q}$ in (24) satisfies the equality very well. The $\beta$ term plays a relatively minor role. In contrast, taking $D_{y y}^{\prime}$ inside the $z$-derivative causes a much larger disagreement. This indicates that the vertical structure of $D_{y y}^{\prime}$ is not negligible. Given the strong similarity between the vertical structure of $D_{y y}^{\prime}$ found here and that reported by Abernathey et al. (2010) for a highly realistic model of the Southern Ocean, it is likely that this issue is relevant for the real ACC.

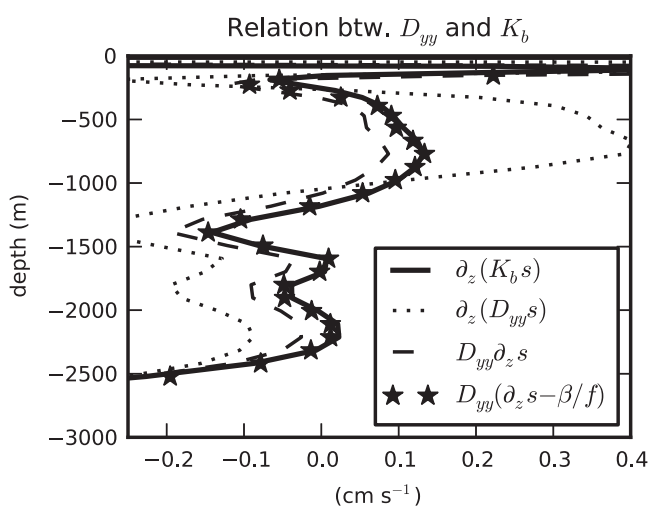

Fig. 15. A test of (24) using $D_{y y}^{\prime}$ in place of $K_{q}$. This illustrates the relationship between the isopycnal mixing rate and the Gent-McWilliams coefficient. Various approximate forms of the equation are also tested. The quantities were evaluated in the center of the domain and were averaged over a meridional width of $200 \mathrm{~km}$. 
We hope that this brief discussion will be noticed by those who wish to translate experimental measurements of isopyncal mixing (for instance, from the DIMES experiment) into values of the GentMcWilliams coefficient for ocean models. Given experimental knowledge of $D_{y y}^{\prime}$, one could proceed by integrating (24) to obtain $K_{b}$, subject to an appropriate boundary condition. We do not pursue this further here, but it is an intriguing topic for future investigation.

\section{Conclusions}

Our paper has not derived any fundamentally new methods; rather, we have unified many different diagnostics of isopycnal mixing and applied them to the same simulation, permitting a side-by-side comparison. We have considered both "perfect" diagnostics, which can realistically only be applied to a numerical model, as well as "practical" diagnostics, which can potentially be applied in field experiments. The results of this comparison are mostly summarized by Fig. 13, which shows appropriately averaged vertical profiles of isopycnal mixing rates as characterized by different diagnostics.

The encouraging conclusion is that these different methods for gauging isopycnal diffusivity produce good agreement. Despite differences in forcing, background state, initial conditions, and gridscale diffusivity, we found mixing rates for passive tracers, QGPV, and Ertel PV with similar magnitude and spatial structure. This spatial structure includes higher mixing rates in the center of the domain, where the eddies are stronger, and, more intriguingly, a distinct mid-depth maximum in the vertical.

We have not gone into great detail on the explanation for this structure, focusing instead on the details of the diagnostic methods themselves; however, the structure is well understood. Most theories for turbulent diffusivity begin with the mixing-length concept of Prandtl (1925) (see, among many, (Green, 1970; Stone, 1972; Held and Larichev, 1996; Stammer, 1998; Smith et al., 2002; Thompson and Young, 2007 [for applications to geostrophic turbulence]). The recent literature contains a growing understanding of the factors responsible for determining the isopycnal mixing rate in the Southern Ocean, and in particular the mid-depth peak. Beginning with Green (1970), linear quasigeostrophic analysis has shown that the QGPV diffusivity must include a mid-depth maximum in unstable eastward flows (see also Killworth, 1997)). The work by Abernathey et al. (2010) showed that such a middepth maxima did exist in a very realistic, eddy-permitting model of the Southern Ocean and attributed its presence to a "critical layer," at which the eddy phase speed equaled the mean flow speed. Further work by Ferrari and Nikurashin (2010) and Klocker et al. (2012a); and Klocker et al., 2012b has confirmed this vertical structure and moved towards a complete theoretical closure for the mixing rates. In the theory of Ferrari and Nikurashin (2010), the competing effects of eddy kinetic energy, eddy size, eddy phase propagation, and zonal mean flow all contribute to the diffusivity. The mid-depth peak was interpreted as a result of strong suppression of mixing by the mean flow at shallower depths.

Our results here, which show that isopycnal mixing rates are consistent across a wide range of diagnostic methods, support the notion that the diffusivity is a fundamental kinematic property of the flow. We hope these results, obtained in a very simplified model, will encourage the community to press on in the effort to measure isopycnal mixing observationally, relate these measurements to theoretical models (such as Ferrari and Nikurashin, 2010), and apply this understanding to improving coarse-resolution models. Indeed efforts are underway to translate the theoretical concepts outlined above into a full-blown eddy closure scheme for ocean models (Bates and Marshall, 2013, manuscript submitted to J. Phys. Oceanogr.).

At the same time, our study indicates some potential pitfalls that might be encountered in attempting to relate observations of isopycnal mixing to diagnostics from numerical models and to theoretical predictions. First of all, there are significant uncertainties associated with practical mixing diagnostics. The errors associated with limited Lagrangian observations are discussed by Klocker et al. (2012b). Here we have also addressed the errors associated with limited isoypcnal tracer release experiments (Section 4.2). Futhermore, there is the problem that both these practical diagnostics involve a spreading-out over large horizontal areas, experiencing different local mixing rates along the way. This spreading means that the measured diffusivities are biased lower than the peak diffusivity at the ACC core (Section 5.1). This smoothing effect means that practical diagnostics are unlikely to be able to detect, for instance, the fine-scale mixing barriers associated with the multiple jets of the ACC (Thompson, 2010).

A final, crucial point is that the diffusivities measured by practical diagnostics can be used directly to estimate the eddy flux of potential vorticity (either the lateral flux of QGPV or the isopycnal flux of Ertel PV). But they can not be employed in a diffusive closure to recover the meridional eddy buoyancy flux below the surface layer in a diffusive buoyancy closure. This is because of the wellknown fact that the buoyancy flux is skew and is therefore not directly related to the isopycnal diffusivity. In other words, the isopycnal diffusivity is not the same as the Gent-McWilliams transfer coefficient. Instead of being equal, the two quantities satisfy (24). While much work remains to be done, we hope our study will help to bridge the gap between observations of isopycnal mixing and the problem of eddy parameterization.

\section{Acknowledgements}

We thank Raffaele Ferrari, Baylor Fox-Kemper, and Malte Jansen, Steve Griffies, and John Marshall for helpful suggestions. We also recognize the substantial improvements to the manuscript that resulted from the comments of three anonymous reviewers.

\section{Appendix A. Errors in the multi-tracer method and $D_{z z}^{\prime}$}

As discussed in detail by Bachman and Fox-Kemper (2013), the multi-tracer method provides a least-squares estimate of the diffusivity tensor $\mathbf{K}$; the method is not exact, and is there is some misfit remaining in the tracer flux that $\mathrm{K}$ cannot explain. The relative errors associated with the method can be quantified by the expression

$\boldsymbol{E}_{n}=\frac{\left|\boldsymbol{F}_{c n}+\mathbf{K} \nabla \bar{c}_{n}\right|}{\left|\boldsymbol{F}_{c n}\right|}$

which gives a component in the $y$ and $z$ directions. Histograms of the relative error of each component are plotted in Fig. 16, including all six tracers at every point in the $(y, z)$ plane (top panel). Evidently, most of the errors are clustered in the bin closest to zero, representing an error of $<2.5 \%$, but significant larger errors exist as well. These errors make it difficult to estimate $D_{z z}^{\prime}$, the minor axis diffusivity, with precision, because the errors are potentially much larger than $D_{z z}^{\prime}$ itself. Also shown in Fig. 16 is the buoyancy flux error

$\boldsymbol{E}_{b}=\frac{\left|\boldsymbol{F}_{b}+\mathbf{K} \nabla \bar{b}\right|}{\left|\boldsymbol{F}_{b}\right|}$,

which characterizes the accuracy of $\mathbf{K}$ for reconstructing the eddy buoyancy flux. (Recall that buoyancy was not used in the inversion for K.) In contrast to Bachman and Fox-Kemper (2013), we find substantially larger errors. This indicates that there are some subtle 

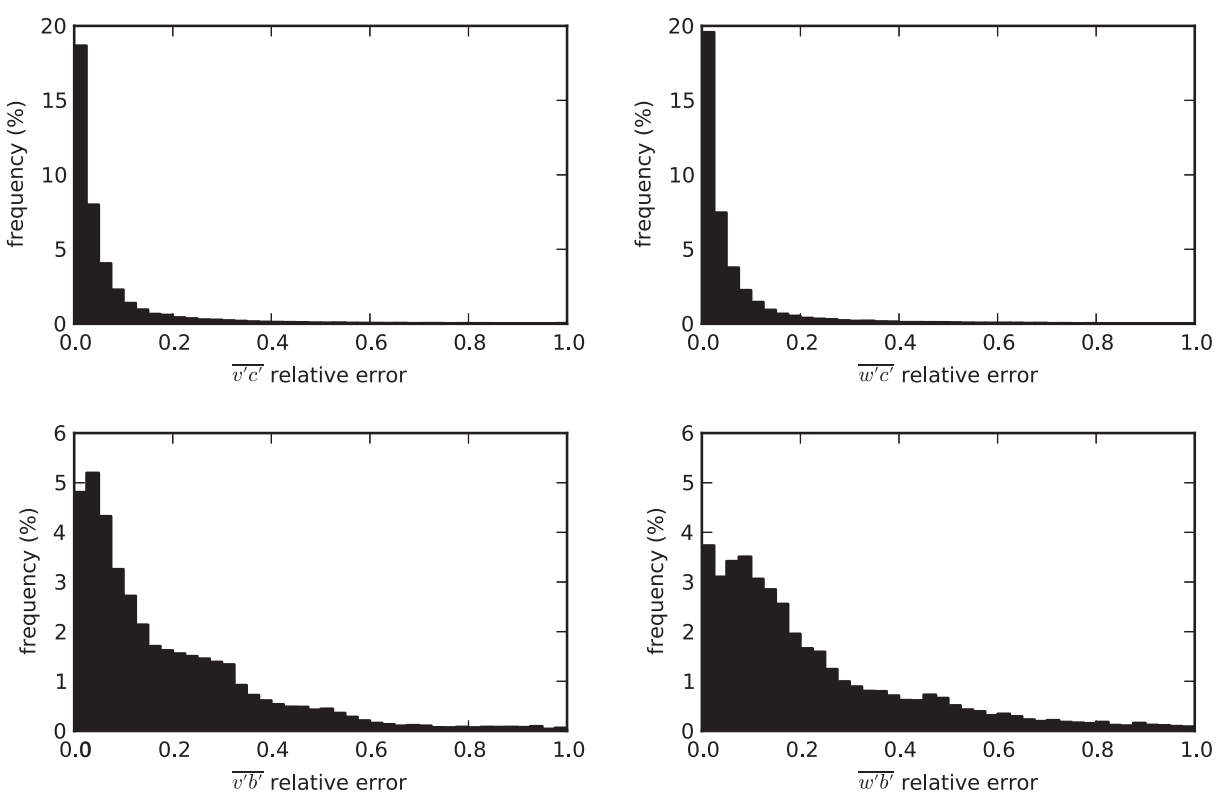

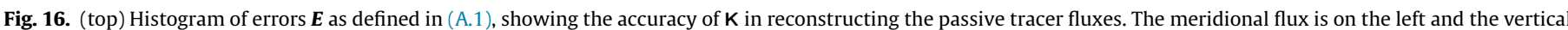
flux is on the right. (bottom) The same error estimate for buoyancy fluxes.

differences in the way buoyancy is transported by eddies compared with the passive tracers. One potential explanation for this is the fact that buoyancy is in a statistical steady state, with forcing at the surface and within the sponge layer, while the tracers experience a transient mixing process.

To illustrate a consequence of the errors in $\mathbf{K}$, consider the formula for $D_{z z}^{\prime}$ in the limit of small mixing angle $\alpha: D_{z z}^{\prime} \simeq D_{z z}-$ $D_{y z}^{2} / D_{y y}$. Typical values of the components of $\mathbf{D}$ are $D_{y y}=5000 \mathrm{~m}^{2} \mathrm{~s}^{-1}, D_{y z}=-5 \mathrm{~m}^{2} \mathrm{~s}^{-1}$, and $D_{z z}=5 \times 10^{-3} \mathrm{~m}^{2} \mathrm{~s}^{-1}$, giving $D_{z z}^{\prime}=0$. Departures from 0 are due to imperfect cancellation between the two terms. As discussed above, the magnitude of the error in $\mathrm{K}$ varies in space and with tracer, but for a rough estimate, let us assume $5 \%$ for each term. This implies that $D_{z z}^{\prime}=0 \pm 6 \times 10^{-4} \mathrm{~m}^{2} \mathrm{~s}^{-1}$.

The numerical model has been constructed to be nearly adiabatic by minimizing the amount of spurious numerical diffusion in the interior. An entire study was devoted to rigorously evaluating the diapycnal diffusion, using both the buoyancy probabilitydensity distribution and passive-tracer based methods (Hill et al., 2012). This study concluded that the model's effective diapycnal diffusivity was less than $10^{-5} \mathrm{~m}^{2} \mathrm{~s}^{-1}$, nearly two orders of magnitude less than the error bounds we estimated for $D_{z z}^{\prime}$.

We now examine Fig. 17 , which plots $D_{z z},-D_{y z}^{2} / D_{y y}$, and $D_{z z}^{\prime}$. This figure shows that $D_{z z}^{\prime}$ is indeed the residual of two much larger terms whose mutual cancellation determines the value of $D_{z z}^{\prime}$. The magnitude of $D_{z z}^{\prime}$ is between $10^{-4}$ and $10^{-3} \mathrm{~m}^{2} \mathrm{~s}^{-1}$. Given the previous results cited above, it seems unlikely that this represents a true diapycnal eddy diffusivity. Rather, it arises both due to the small errors inherent in $\mathbf{K}$ and from time dependence of the tracer concentrations. As shown definitively by Hill et al. (2012), buoyancy is subjected to a much smaller rate of diapycnal diffusion than is suggested by $D_{z z}^{\prime}$, which seems to suggest that $D_{z z}^{\prime}$ is not a very meaningful quantity here. Certainly it does not contribute strongly to the tracer transport.

On the other hand, $D_{y y}^{\prime} \simeq D_{y y}$, with no cancellation between large competing terms. This means that the errors of $1-5 \%$ in $\mathrm{K}$ shown in Fig. 16 apply in a straightforward way to $D_{y y}^{\prime}$.

\section{Appendix B. Tracer release experiments}

As discussed in Hill et al. (2012), mimicking tracer release experiments in an ocean model can be problematic. One wants the initial tracer distribution to be as compact as possible (to be close to an isopycnal) but not too small compared to the grid scale. Also, the initial distribution has to be small enough, relative to the domain, to leave ample time before the tracer is transported into the surface mixed layer or north/south boundaries.

As a compromise (following Hill et al. (2012)), the tracer field is initialized with a 3D Gaussian shape with $50 \mathrm{~m}$ vertical and $5 \mathrm{~km}$ horizontal half-width. The tracer has a maximum value of one. We carried out 16 releases at 11 depths (shown by the open circles in Fig. 11). Each set of 16 releases consists of eight releases, $125 \mathrm{~km}$
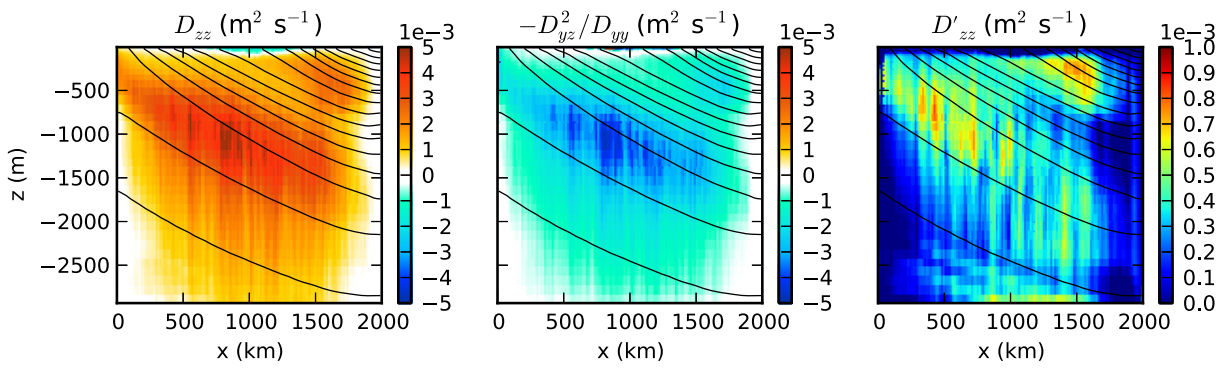

Fig. 17. The terms of the tensor $\mathbf{D}$ relevant for estimating $D_{z z}^{\prime}$. The third panel is approximately given by the sum of the first two panels. Note the different color scales. 


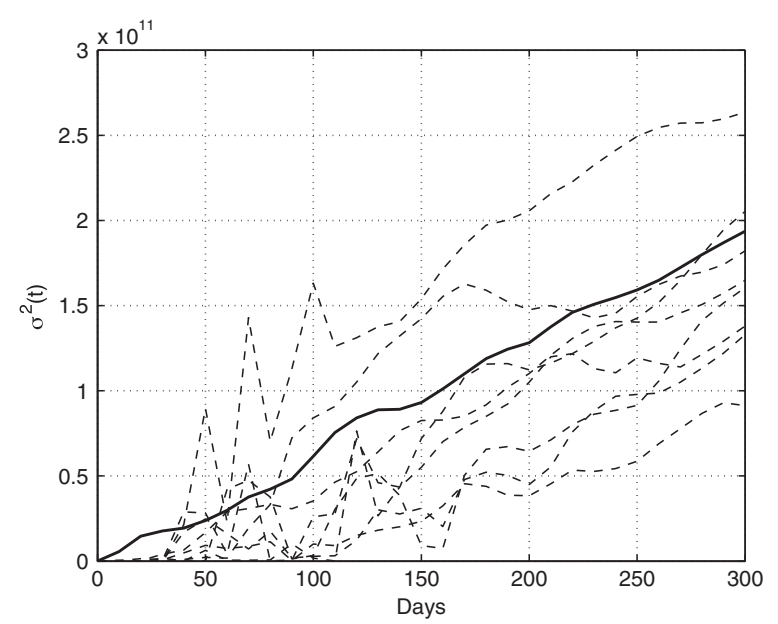

Fig. 18. Time evolution of $\sigma^{2}$ (in $\mathrm{m}^{2}$ ) for each individual tracer release experiment (dashed lines) and for the 16-member ensemble (thick solid line) at $1200 \mathrm{~m}$ depth. Linear growth with time signifies a constant diffusivity.

apart along the central axis of the channel followed by a second set of eight 300 days later. The 3D tracer distributions are sampled every 10 days for 300 days. In order to calculate the isopycnal diffusivity, all vertical profiles are first plotted around a relative vertical coordinate centered on the target temperature of the release and then integrated vertically and zonally to produce a meridional profile. A Gaussian curve is fitted to the reconstructed meridional profile (from a single tracer or averaged from an ensemble of profiles, see examples in Fig. 10, bottom panels). The best-fit halfwidth $\sigma_{y}(t)$ relates to the effective diffusivity through:

$K_{\mathrm{I}}=\frac{1}{2} \frac{d \sigma_{y}^{2}}{d t}$.

Fig. 18 illustrates the time evolution of $\sigma_{y}(t)$ for a few single tracers (dashed lines) and for the 16-member ensemble mean (thick solid) for releases at $1200 \mathrm{~m}$ depth. The initial behavior of sigma is rather erratic for individual tracers, but often approach a linear tendency after 150 days. The ensemble mean value is very nearly linear from the tracer release onward. Note that this is not true at all depths-in some cases the ensemble mean value only settles down into a linear trend after a 100 days. For consistency, all isopycnal diffusivities shown here are obtained by a best linear fit of $\sigma^{2}(t)$ between 1500 and 300 days.

Although $\sigma_{y}(t)$ from individual tracers exhibits rather similar trends after $\sim 150$ days, the differences in slopes are sufficient to result in large uncertainties on $K_{I}$, as much as $\pm 1500 \mathrm{~m}^{2} \mathrm{~s}^{-1}$ at $1200 \mathrm{~m}$.

\section{References}

Abernathey, R., Marshall, J., Shuckburgh, E., Mazloff, M., 2010. Enhancement of mesoscale eddy stirring at steering levels in the Southern Ocean. J. Phys. Oceanogr., 170-185.

Abernathey, R., Marshall, J., Ferreira, D., 2011. The dependence of Southern Ocean meridional overturning on wind stress. J. Phys. Oceanogr. 41 (12), 2261-2278.

Andrews, D., Holton, J., Leovy, C., 1987. Middle Atmosphere Dynamics. Academic Press.

Bachman, S., Fox-Kemper, B., 2013. Eddy parameterization challenge suite I: Eady spindown. Ocean Modelling 64, 12-18.

Bratseth, A.M., 1998. On the estimation of transport characteristics of atmospheric data sets. Tellus 50A, 451-467.

Cerovecki, I., Marshall, J., 2008. Eddy modulation of air-sea interaction and convection. J. Phys. Oceanogr. 38, 65-93.

Davis, R., 1985. Drifter observations of coastal surface currents during CODE: the statistical and dynamical views. J. Geophys. Res. 90, 4756-4772.

Davis, R., 1991. Observing the general circulation with floats. Deep Sea Res. 38A, S531-S571.
Davis, R., 1994. Lagrangian and Eulerian measurements of ocean transport processes. Ocean Processes in Climate Dynamics: Global and Mediterranean Examples. Springer, pp. 29-60.

Eden, C., 2010. Parameterising meso-scale eddy momentum fluxes based on potential vorticity mixing and a gauge term. Ocean Modelling 32, 58-71.

Ferrari, R., Nikurashin, M., 2010. Suppression of eddy diffusivity across jets in the Southern Ocean, J. Phys Oceanogr. 40, 1501-1519.

Gent, P., McWilliams, J., 1990. Isopycnal mixing in ocean circulation models. J. Phys. Oceanogr. 20, 150-155.

Gent, P., Willebrand, J., McDougal, T., McWilliams, J., 1995. Parameterizing eddyinduced tracer transports in ocean circulation models. J. Phys. Oceanogr. 25 463-475.

Gille, S.T., Ledwell, J., Naveira Garabato, A., Speer, K., Balwada, D., Brearley, A., Girton, J.B., Griesel, A., Ferrari, R., Klocker, A., LaCasce, J., Lazarevich, P., Mackay, N., Meredith, M.P., Messias, M.-J., Owens, B., Sallée, J.-B., Sheen, K., Shuckburgh, E. Smeed, D.A., Laurent, L.C.S., Toole, J.M., Watson, A.J., Wienders, N., Zajaczkovski, U., 2012. The diapycnal and isopycnal mixing experiment: a first assessment. CLIVAR Exchanges 17 (1), 46-48.

Green, J.S.A., 1970. Transfer properties of the large-scale eddies and the general circulation of the atmosphere. Quart. J. Roy. Meteor. Soc. 96, 157-185.

Griffies, S.M., 1998. The Gent-McWilliams skew flux. J. Phys. Oceanogr. 28, 831841.

Haynes, P., Shuckburgh, E., 2000a. Effective diffusivity as a diagnostic of atmospheric transport. Part I: stratosphere. J. Geophys. Res 105, 22777-22794.

Haynes, P., Shuckburgh, E., 2000b. Effective diffusivity as a diagnostic of atmospheric transport. Part II: Troposphere and lower stratosphere. J. Geophys. Res 105, 795-810.

Held, I.M., Larichev, V.D., 1996. A scaling theory for horizontally homogeneous, baroclinically unstable flow on a beta plane. J. Atmos. Sci. 53 (7), 946-953.

Hill, C., Ferreira, D., Campin, J.-M., Marshall, J., Abernathey, R., Barrier, N., 2012. Controlling spurious diapycnal mixing in eddy-resolving height-coordinate ocean models: Insights from virtual deliberate tracer release experiments. Ocean Modelling 45-46, 14-26.

Ito, T., Marshall, J., 2008. Control of lower-limb overturning circulation in the Southern Ocean by diapycnal mixing and mesoscale eddy transfer. J. Phys. Oceanogr. 38, 2832-2845.

Jansen, M., Ferrari, R., 2013. The vertical structure of the eddy diffusivity and the equilibrarion of the extra-tropical atmosphere. J. Atmos Sci. 70, 1456-1469.

Killworth, P.D., 1997. On the parameterization of eddy transfer. Part I: Theory. J. Marine Res. 55, 1171-1197.

Klocker, A., Ferrari, R., LaCasce, J.H., 2012a. Estimating suppression of eddy mixing by mean flow. J. Phys. Oceanogr. 9, 1566-1576.

Klocker, A., Ferrari, R., LaCasce, J.H., Merrifield, S.T., 2012b. Reconciling float-based and tracer-based estimates of eddy diffusivities. J. Marine Res. 70 (4) 569-602.

Kuo, A., Plumb, R.A., Marshall, J., 2005. Transformed Eulerian-mean theory. Part II: Potential vorticity homogenization and equilibrium of a wind- and buoyancydriven zonal flow. J. Phys. Oceanogr. 45, 175-187.

LaCasce, J.H., 2008. Statistics from lagrangian observations. Progr. Oceanography 77, $1-29$.

Lauderdale, J.M., Naveira Garabato, A.C., Oliver, K.I.C., Follows, M.J., Williams, R.G., 2013. Wind-driven changes in Southern Ocean residual circulation, ocean carbon reservoirs and atmospheric CO2. Clim. Dyn., 1-20.

Ledwell, J.R., Bratkovich, A., 1995. A tracer study of mixing in the santa cruz basin. J. Geophys. Res. 100 (C10), 20681.

Ledwell, J.R., Watson, A.J., Law, C.S., 1998. Mixing of a tracer in the pycnocline. J. Geophys. Res. 103 (C10), 21,499-21,592.

Ledwell, J.R., St. Laurent, L.C., Girton, J.B., 2011. Diapycnal mixing in the antarctic circumpolar current. J. Phys. Oceanogr. 41, 241-246.

Liu, C., Köhl, A., Stammer, D., 2012. Adjoint based estimation of eddy induced tracer mixing parameters in the global ocean. J. Phys. Oceanogr. 42, 1186-1206.

Lu, J., Speer, K., 2010. Topography, jets, and eddy mixing in the Southern Ocean. J. Marine Res. 68, 479-502.

Lumpkin, R., Speer, K., 2007. Global meridional overtuning. J. Phys. Oceanogr. 37, 2537-2550.

Marshall, J., Radko, T., 2003. Residual mean solutions for the antarctic circumpolar current and its associated overturning circulation. J. Phys. Oceanogr. 33, $2341-$ 2354.

Marshall, J., Shutts, G., 1981. A note on rotational and divergent eddy fluxes. J. Phys. Oceanogr. 21, 1677-1681.

Marshall, J., Speer, K., 2012. Closing the meridional overturning circulation through Southern Ocean upwelling. Nature Geosci. 5, 171-180.

Marshall, J., Adcroft, A., Hill, C., Perelman, L., Heisey, C., 1997. A finite-volume, incompressible Navier Stokes model for studies of the ocean on parallel computers. J. Geophys. Res. 102, 5753-5766.

Marshall, J., Hill, C., Perelman, L., Adcroft, A., 1997. Hydrostatic, quasi-hystrostatic, and non-hydrostatic ocean modeling. J. Geophys. Res. 102, 5733-5752.

Marshall, J., Shuckburgh, E., Jones, H., Hill, C., 2006. Estimates and implications of surface eddy diffusivity in the Southern Ocean derived from tracer transport. J. Phys. Oceanogr. 36, 1806-1821.

McIntosh, P.C., McDougall, T.J., 1996. Isopycnal averaging and the residual mean circulations. J. Phys. Oceanogr. 26, 1655-1661.

Nakamura, N., 1996. Two-dimensional mixing, edge formation, and permeability diagnosed in an area coordinate. J. Atmos. Sci. 53, 1524-1537.

Nakamura, N., 2001. A new look at eddy diffusivity as a mixing diagnostic. J. Atmos, Sci. 58, 3685-3702. 
Nakamura, N., 2008. Quantifying inhomogeneous, instantaneous, irreversible transport using passive tracer field as a coordinate. Lecture Notes Phys. 744 137-164.

Nakamura, N., Ma, J., 1997. Modified lagrangian-mean diagnostics of the stratospheric polar vortices 2 . Nitrous oxide and seasonal barrier migration in the cryogenic limb array etaion spectrometer and skyhi general circulation model. J. Geophys. Res. 102 (25), 721-725, 735.

Naveira-Garabato, A.R., Ferrari, R., Polzin, K., 2011. Eddy stirring in the Southern Ocean. J. Geophys. Res. 116, C09019.

Nikurashin, M., Vallis, G., 2012. A theory of the interhemispheric meridional overturning circulation and associated stratification. J. Phys. Oceanogr. 42, $1652-1667$.

Plumb, R.A., 1979. Eddy fluxes of conserved quantities by small-amplitude waves. J. Atmos. Sci. 36, 1699-1705.

Plumb, R.A., Ferrari, R., 2005. Transformed eulerian-mean theory. Part I: Nonquasigeostrophic theory for eddies on a zonal-mean flow. J. Phys. Oceanogr. 35, 165-174.

Plumb, R.A., Mahlman, J.D., 1987. The zonally-averaged transport characteristics of the gfdl general circulation/tracer model. J. Atmos. Sci. 44, 298-327.

Prandtl, L., 1925. Bericht untersuchungen zur ausgebildeten turbulenz. Zs. angew. Math. Mech. 5, 136-139.

Prather, M.J., 1986. Numerical advection by conservation of second-order moments. J. Geophys. Res. 91 (D6), 6671-6681.

Redi, M., 1982. Oceanic isopycnal mixing by coordinate rotation. J. Phys. Oceanogr. $12,1154-1158$.

Rhines, P.B., Young, W.R., 1982. Homogenization of potential vorticity in planetary gyres. J. Fluid Mech. 122, 347-367.

Sallée, J.B., Speer, K., Morrow, R., Lumpkin, R., 2008. An estimate of Lagragian eddy statistics and diffusion in the mixed layer of the Southern Ocean. J. Marine Res. 66 (4), 441-463.

Shuckburgh, E., Jones, H., Marshall, J., Hill, C., 2009a. Robustness of effective diffusivity diagnostic in oceanic flows. J. Phys. Oceanogr. 39, 1993-2009.
Shuckburgh, E., Jones, H., Marshall, J., Hill, C., 2009b. Understanding the regional variability of eddy diffusivity in the pacific sector of the Southern Ocean. J. Phys. Oceanogr. 39, 2011-2023.

Shuckburgh, E., Maze, G., Ferreira, D., Marshall, J., Jones, H., Hill, C., 2011. Mixed layer lateral eddy fluxes mediated by air-sea interaction. J. Phys. Oceanogr. 41, 130-144.

Smith, K.S., Marshall, J., 2009. Evidence for enhanced eddy mixing at mid-depth in the Southern Ocean. J. Phys. Oceanogr. 39, 50-69.

Smith, K.S., Boccaletti, G., Henning, C.C., Marinov, I., Tam, C.Y., Held, I.M., Vallis, G.K., 2002. Turbulent diffusion in the geostrophic inverse cascade. J. Fluid Mech. 469, $13-48$.

Stammer, D., 1998. On eddy characteristics, eddy transports, and mean flow properties. J. Phys. Oceanogr. 28, 727-739.

Stone, P.H., 1972. A simplified radiative-dynamical model for the static stability of rotating atmospheres. J. Atmos. Sci. 29 (3), 405-417.

Taylor, G.I., 1921. Diffusion by continuous movements. Proc. London Math. Soc. s220, 196-212.

Thompson, A.F., 2010. Jet formation and evolution in baroclinic turbulence with simple topography. J. Phys. Oceanogr. 40, 257-274

Thompson, A.F., Young, W.R., 2007. Two-layer baroclinic eddy heat fluxes: zonal flows and energy balance. J. Atmos. Sci. 64, 3214-3232.

Treguier, A.M., 1999. Evaluating eddy mixing coefficients from eddy-resolving ocean models: A case study. J. Marine Res. 57, 89-108.

Treguier, A.M., Held, I., Larichev, V., 1997. Parameterization of quasigeostrophic eddies in primitive equation ocean models. J. Phys. Oceanogr. 27, 567-580.

Vallis, G., 2006. Atmospheric and Oceanic Fluid Dynamics. Cambridge University Press.

Wilson, C., Williams, R.G., 2004. Why are eddy fluxes of potential vorticity difficult to parameterize? J. Phys. Oceanogr. 34, 142-155.

Winters, K., D’Asaro, E., 1996. Diascalar flux and the rate of fluid mixing. J. Fluid Mech. 317, 179-193. 